NIKHEF 97-015

hep-ph/9704203

\title{
Charm production in deep inelastic and diffractive scattering
}

\author{
L.P.A. Haakman ${ }^{a}$, A.B. Kaidalov ${ }^{b}$, J.H. Koch ${ }^{a}$ \\ ${ }^{a}$ National Institute for Nuclear Physics and High Energy Physics (NIKHEF), \\ P.O. Box 41882, NL-1009 DB Amsterdam, The Netherlands \\ ${ }^{b}$ Institute of Theoretical and Experimental Physics, \\ B. Cheremushinskaya 25, 117259 Moscow, Russia
}

(March 1997)

\begin{abstract}
We consider the production of charm by real and virtual photons. Special attention is paid to diffractive charm production, which provides information on the gluonic content of the Pomeron. Our calculations are based on the gluon distributions of the CKMT-model, which is shown to lead to agreement with the data on open charm production in deep inelastic scattering. We compare predictions for diffractive charm production of different models for the distribution of gluons in the Pomeron. Experiments at HERA should be able to discriminate between them. Predictions for beauty production in diffractive and non-diffractive interactions of photons are also given.
\end{abstract}

\section{INTRODUCTION}

The production of heavy quarks in deep inelastic electron scattering from a proton has received increasing attention recently, since this reaction is seen as a tool to probe the gluon distribution in the nucleon. Due to the large mass of the quark, the reaction is believed to be driven by a perturbative mechanism, photon-gluon fusion, and is therefore sensitive to the nucleonic gluon density. We consider here in a consistent way the production of charm in deep inelastic scattering (DIS) as well as in hard diffractive scattering. The latter process, while driven by the same basic reaction mechanism, probes a different gluon density. As the diffractive processes are mostly seen as mediated by the Pomeron exchange, the relevant quantity in diffractive production is then the gluon distribution in the Pomeron; a review can be found in Ref. [1].

The distribution of gluons in the proton is known now comparatively well from deep inelatic scattering for $x \geq 10^{-2}$, but for smaller values of $x$ information on $g_{p}\left(x, Q^{2}\right)$ is very limited. A comparison of theoretical predictions on charm production in the HERA energy range with experimental data allows one to test small$x$ behaviour of gluonic distributions.

Diffractive production of hadrons in deep inelastic scattering has been observed in experiments at HERA [2,3]. The gluonic content of the Pomeron is poorly known. Studies of the $Q^{2}$-dependence of the Pomeron structure function [4 8] lead to the conclusion that the distribution of gluons in the Pomeron is hard and that they carry the main part of the Pomeron momentum. However there are big differences between gluon distribution functions $g_{P}\left(x, Q^{2}\right)$ in different models.

It is the purpose of this paper to present a consistent approach to open charm photo- and electroproduction as well as diffractive charm production. The gluon distributions we use are based on the model of Ref. [9], hereafter referred to as CKMT. No new parameters are introduced into our calculations. In this 
approach there is only one basic Pomeron, which contributes in different ways to both "soft" and "hard" mechanisms. This can be represented in terms of an effective or "dressed" Pomeron with a $Q^{2}$-dependent intercept. It was the basis of a very compact parametrization by CKMT for the structure function $F_{2}$ and distributions of quarks and gluons in the proton, which was used as an initial condition for QCD evolution [9]. Also diffractive vector meson production $(\rho, \phi, \psi)$ by real and virtual photons [10] could be described very well by this model without additional parameters.

There have been a variety of investigations on aspects of charm production in deep inelastic and diffractive scattering, in part going beyond the leading order. In order to achieve a clear and simple discussion of these reactions, we confine ourselves to the leading order terms in the cross sections. This more phenomenological approach seems reasonable in view of the large sensitivity of perturbative QCD calculations to the heavy quark mass and the present uncertainty in the data.

In Section 2 we shall first consider inclusive charm photo- and electroproduction on the proton. We shall formulate a method of calculation of the cross sections of heavy quark production for arbitrary values of $Q^{2}$. It will be shown that the gluon distribution proposed by CKMT gives a very good description of the existing data on charm production. We therefore proceed in Section 3 to calculate charm production in diffractive scattering in the context of the CKMT model and compare to other models. In Section 4 we present predictions for inelastic and diffractive beauty production by real and virtual photons.

\section{CHARM PHOTO- AND ELECTROPRODUCTION}

In this section we consider the open charm production by real or virtual photons on a proton. In the past several prescriptions have been proposed to incorporate the contribution of charm into $F_{2}\left(x, Q^{2}\right)$. A widely used method [1] 14 was to generate the charm quarks dynamically, starting with no intrinsic charm below some threshold $Q_{t h}^{2} \sim m_{c}^{2}$ and to produce charm quarks (considered as massless) through QCD evolution. However this results into a too large charm contribution in the threshold region. We therefore choose a different approach. For moderate $Q^{2}$, the fact that the mass of the charm quark is comparatively large, $m_{c} \sim 1.5 \mathrm{GeV} \gg \Lambda_{Q C D}$, makes it possible to apply perturbation theory. It was shown in Ref. [15] that therefore more realistic predictions for charm production can be obtained from the photon-gluon fusion diagram of Fig. 1a. This is true in a broad region of $Q^{2}$. Next to leading order (NLO) calculations in QCD perturbation theory have been carried out [15 22] for heavy quark production. These higher order terms lead to no new qualitative feature and can be incorporated by adjusting the parameters of the lowest order calculation [15. In view of this perturbative stability and since there are uncertainties in the charm quark mass and the factorization scale, we prefer to simply work in the leading order (LO), where the calculations are very transparent. We shall show that by properly chosing the charm quark mass and factorization scale one obtains a good description of the data for charm production in DIS. For $Q^{2} \gg m_{c}^{2}$, on the other hand, higher order diagrams of the QCD perturbation theory should be resummed [23]. Since mass effects can then be neglected, the charm quarks will be produced dynamically through QCD evolution, where the charmed quark is considered as massless. We obtain the charm quark distribution needed as input for this evolution through the photon-gluon fusion mechanism.

At moderate $Q^{2}$, the main contribution for large centre-of-mass energies $\sqrt{s}$ is expected to come from the gluon fusion process, shown in Fig. 1a, where a gluon from the proton interacts with the photon and produces a charm anti-charm quark pair. We will discuss this mechanism first. Contributions from "resolved" charm production, Fig. 1b, are less important and will be dealt with later.

In QCD perturbation theory the cross section for the process $\gamma^{(*)} p \rightarrow c \bar{c} X$ can be written as a convolution of the gluon distribution $g_{p}\left(z, \mu_{f}^{2}\right)$ and the partonic cross section of the photon-gluon fusion process $\hat{\sigma}(\gamma g \rightarrow c \bar{c})$,

$$
\sigma\left(\gamma^{*} p \rightarrow c \bar{c} X\right)=\int_{z_{m i n}}^{1} d z \hat{\sigma}_{\gamma g \rightarrow c \bar{c}}\left(x, z, \frac{Q^{2}}{m_{c}^{2}}, m_{c}^{2}\right) g_{p}\left(z, \mu_{f}^{2}\right)
$$


The threshold condition for production af a charm-anticharm pair leads to a lower bound for the fraction $z$ of the proton momentum carried by the gluon, $z_{\min }=a x$, where $a=1+4 m_{c}^{2} / Q^{2}$ and $x$ is the Bjorken variable. For the partonic cross section of charm production by photon-gluon fusion, one finds [23, 24] with the Hand convention for the flux

$$
\hat{\sigma}_{\gamma g \rightarrow c \bar{c}}\left(x, z, \frac{Q^{2}}{m_{c}^{2}}, m_{c}^{2}\right)=\frac{4 \pi^{2} \alpha_{E M}}{Q^{2}(1-x)} 2 x e_{c}^{2} \frac{\alpha_{s}\left(\mu_{F}^{2}\right)}{2 \pi} C\left(\frac{x}{z}, \frac{m_{c}^{2}}{Q^{2}}\right),
$$

where $\mu_{f}$ is the factorization scale and $e_{c}$ the charge of the charm quark in units of $e$. In leading order the coefficient function $C$ is

$$
\begin{aligned}
C(\zeta, r)= & \frac{1}{2}\left[\zeta^{2}+(1-\zeta)^{2}+4 \zeta(1-3 \zeta) r-8 \zeta^{2} r^{2}\right] \ln \frac{1+v}{1-v} \\
& +\frac{v}{2}[-1+8 \zeta(1-\zeta)-4 \zeta(1-\zeta) r]
\end{aligned}
$$

with

$$
v^{2}=1-\frac{4 r \zeta}{1-\zeta}
$$

In the limit $Q^{2}=0$ Eq.(1) yields the photoproduction cross section. The contribution of charm production to the proton structure function is given by

$$
F_{2}^{c \bar{c}}\left(x, Q^{2}\right)=\frac{Q^{2}(1-x)}{4 \pi^{2} \alpha_{E M}} \sigma\left(\gamma^{*} p \rightarrow c \bar{c} X\right)
$$

For the gluon distribution, a crucial ingredient in the calculation of the cross section, we use the CKMT-parametrization of Ref. [9]. It gives a good description of the HERA data for the proton structure function $F_{2}\left(x, Q^{2}\right)$ at all $Q^{2}$ and for diffractive vector meson production [9, 10. We briefly summarize here only the physics background; details can be found in the Appendix and in Ref. [9].

Experimental studies of small $x$ DIS at HERA 25,26 had shown a fast increase of the proton structure function $F_{2}\left(x, Q^{2}\right)$ as $x \rightarrow 0$, which was considered by some authors as an evidence for a "hard" Pomeron. This Pomeron has an intercept $\alpha_{P}(0)$ substantially above unity, contrary to the "soft" Pomeron, which is observed in high-energy hadronic interactions and photoabsorption, which has an intercept only slightly above 1: $\alpha_{P}(0) \equiv \Delta+1 \approx 1.08$. It was argued however by CKMT in Ref. [9] that this $\alpha_{P}(0)$ extracted from high-energy behaviour of hadronic total cross sections is not the actual intercept of the Pomeron itself, but an effective value that incorporates large effects of Pomeron rescattering (multi-Pomeron cuts) in soft processes. The actual "bare" value of the pole intercept extracted from the analysis of many features of hadronic interactions, taking into account multi-Pomeron processes, was found to be substantially higher, corresponding to $\Delta \approx 0.2$ 27. At large $Q^{2}$ in DIS the contributions from rescatterings are much smaller than in hadronic interactions (or $\gamma p$ ) and the "bare" intercept determines the behaviour of structure functions at $Q^{2}>1 \mathrm{GeV}^{2}$. In this approach there is thus only one Pomeron, which contributes to both "soft" and "hard" processes. This effective or "dressed" Pomeron then has a $Q^{2}$-dependent intercept. It was used by CKMT to provide a compact parametrization of the structure function $F_{2}$ and the distributions of quarks and gluons in the nucleon. These distributions are used as initial condition for the QCD evolution [9].

The gluon distribution resulting from this model is

$$
x g_{p}\left(x, Q^{2}\right)=C_{g} x^{-\Delta\left(Q^{2}\right)}(1-x)^{n\left(Q^{2}\right)+3}
$$

with the effective Pomeron intercept determined by 


$$
\Delta\left(Q^{2}\right)=\Delta(0)\left(1+\frac{d_{0} Q^{2}}{Q^{2}+d_{1}}\right)
$$

For low $x$ the gluon density in this model exhibits the $x$ dependence which is characteristic for the Pomeron exchange. The behaviour at $x \sim 1$ has been obtained from counting rules for the sea quarks which are a factor $1-x$ softer than the gluons. Finally, the factor $C_{g}$ follows from the momentum sum rule. This parametrization is valid up to $Q^{2}=5 \mathrm{GeV}^{2}$. We actually use it only up to $2 \mathrm{GeV}^{2}$ and for higher values QCD evolution is applied where the parametrization provides the initial condition. We take the running coupling constant with $\Lambda=0.20 \mathrm{GeV}$ and four flavours.

The results for charm production are very sensitive to small changes in the value of the charm mass. We will use charm photoproduction to fix the value of this parameter. In Eqs.(11) and (2) one encounters the factorization scale $\mu_{f}$. The NLO results in Ref. [15] showed that the scale $\mu_{f}^{2}=4 m_{c}^{2}$ yields the best stability of the perturbative calculations and we will use this now for photoproduction; other choices will be discussed below in connection with electroproduction. Since this factorization scale is above the value of $Q^{2}$ for which the parametrization is valid, we use the leading order QCD evolution equations for three flavours to obtain the gluon distribution at $\mu_{f}^{2}$.

We calculated open charm production corresponding to the diagram of Fig. 1a with the gluon distribution function as given in (6). The results for the total inclusive cross section are shown for four different values of the charm mass in Fig. 2. One can see that relatively small changes in the charm mass lead to large differences in the cross section. For a charm quark mass $m_{c}=1.4 \mathrm{GeV}$ our curves are close to the experimental data and look similar to the results of the NLO calculation [15,22]. In the following we shall use this value of the charm quark mass.

For very large energies $\sqrt{s}$ the resolved contribution, shown in Fig. 1b, starts to be important. In this production mechanism a gluon from the photon interacts with a gluon from the proton to produce a charm anti-charm pair. To obtain the contribution from the resolved production, we therefore need the gluon distribution in the photon, $g_{\gamma}\left(x, Q^{2}\right)$. This distribution is poorly known at present. For an estimate of the resolved contribution, we use a distribution obtained by the same method as for the determination of the gluon distribution of the proton in Ref. [9] and thus introduce no new parameters. At small $x$ factorization takes place in the Regge pole model. Therefore the gluon distribution in the photon at low $x$ is proportional to that of the proton; for this proportionality factor we find

$$
e_{p}^{\gamma} \equiv \lim _{x \rightarrow 0} \frac{g_{\gamma}\left(x, Q^{2}\right)}{g_{p}\left(x, Q^{2}\right)}=\lim _{s \rightarrow \infty} \frac{\sigma_{\gamma p}^{t o t}}{\sigma_{p p}^{t o t}} \approx 0.003 .
$$

This factorization is approximately valid also when Pomeron cuts are taken into account. The behaviour in the limit $x \rightarrow 1$ is different for the photon and proton and can be found from counting rules. So we write $g_{\gamma}\left(x, Q^{2}\right)$ in the form

$$
x g_{\gamma}\left(x, Q^{2}\right)=e_{p}^{\gamma} \frac{x g_{p}\left(x, Q^{2}\right)}{(1-x)^{2}}=e_{p}^{\gamma} C_{g} x^{-\Delta\left(Q^{2}\right)}(1-x)^{n\left(Q^{2}\right)+1}
$$

The contribution of the diagram in Fig. $1 \mathrm{~b}$ is shown in Fig. 2. It is negligible at energies up to $\sqrt{s} \sim 10 \mathrm{GeV}$, but increases with energy faster than the dominant photon-gluon fusion contribution of Fig. 1a. This contribution is of the order of $10 \%$ already at HERA energies. For other models of $g_{\gamma}\left(x, Q^{2}\right)$ this contribution is larger [22]. It is also sensitive to the value of the factorization scale and increases for smaller values of this scale.

We now turn to electroproduction. First we will consider the factorization scale $4 m_{c}^{2}$ as in photoproduction. The production of charm quarks give a large contribution to the DIS structure function $F_{2}\left(x, Q^{2}\right)$ at small $x$ and large $Q^{2} \gg m_{c}^{2}$, where they can be considered in the framework of QCD evolution equation as massless. However at $Q^{2} \sim 10 \mathrm{GeV}^{2}$ or less the charm mass is very important and the charm quarks 
cannot be treated in the same way as the light quarks [15]. We therefore deal with electroproduction in two different ways, depending on the value of $Q^{2}$. For values of $Q^{2}$ less than a certain value $\overline{Q^{2}}$, we use the perturbative approach for the gluon fusion process with massive charm quarks as given by Eq.(11). To obtain the gluon distribution at the factorization scale $4 m_{c}^{2}$, we proceed as in the photoproduction case; we start the QCD evolution for three massless flavours from $Q_{0}^{2}=2 \mathrm{GeV}^{2}$. With this distribution, the charm contribution is directly obtained from Eq.(2). For virtualities larger than $\overline{Q^{2}}$ the charm quarks are produced through massless QCD evolution with four flavours. For the input distributions, we use the light quark distributions as obtained above from three flavour massless QCD evolution up to $\overline{Q^{2}}$. The charm input distribution is generated by photon-gluon fusion at $\overline{Q^{2}}$.

We determine the transition value $\overline{Q^{2}}$ from the perturbative approach with massive charmed quarks to the massless QCD evolution by demanding that this procedure creates an $F_{2}^{c \bar{c}}$ below and above $\overline{Q^{2}}$ with a smooth derivative with respect to $Q^{2}$ at this point. We found that the values of $\overline{Q^{2}}$ yielding a smooth transition in the region of small $x$ vary from $30 \mathrm{GeV}^{2}$ to $100 \mathrm{GeV}^{2}$. In Fig. 3 a we show the charm contribution to the proton structure function, $F_{2}\left(x, Q^{2}\right)$, as given by Eq.(2) as a function of the virtuality $Q^{2}$ at different values of $x$; the transition from massive to massless treatment of the charm quarks is made at $50 \mathrm{GeV}^{2}$. We also show the logarithmic derivatives in Fig. $3 \mathrm{~b}$ to see their discontinuities at $\overline{Q^{2}}$. The change of $\Lambda$ in the running coupling constant at the charm threshold has been taken into account. It follows from Figs. 3a and 3b that for very small $x\left(x \leq 10^{-3}\right)$ the transition from one regime to the other is very smooth and for $Q^{2}>50 \mathrm{GeV}^{2}$ charmed quarks can safely be considered as massless in the QCD evolution equations. This is in agreement with results obtained in Ref. [19] and also with Ref. [15], where it has been stated that for $W^{2}=Q^{2}(1-x) / x \leq 10^{6} \mathrm{GeV}^{2}$ the gluon fusion model should be applied. For $x \sim 0.1$ the mass effects are important up to much larger values of $Q^{2}$. Thus, from Fig. 3a we can conclude that for the small $x$ and $Q^{2} \lesssim 500 \mathrm{GeV}^{2}$ the difference between the prediction of the gluon-photon fusion diagram and its QCD-evolved contribution is small.

So far, we have used the factorization scale $4 m_{c}^{2}$. Another natural candidate for electroproduction is $4 m_{c}^{2}+Q^{2}$. To examine this possibility, we show in Fig. 3c and 3d the analogous results for this scale. The features are quite different. Now for large $x$ the discontinuity is small, but for small $x$ it is large, just the reverse of what one finds for the constant factorization scale. This suggests that one could use a different factorization scale and/or different transition value $\overline{Q^{2}}$ for different kinematical regimes to obtain a smooth transition from massive to massless quarks.

Finally, in Fig. 4 we make a comparison of our predictions for charm photo- and electroproduction with the data. We show results as a function of $W=\sqrt{s}$ for the factorization scales $4 m_{c}^{2}$ and $4 m_{c}^{2}+Q^{2}$; for the latter we only show predictions at high $Q^{2}$ where the difference to the fixed scale is large. Note that the cross sections for the different values of $Q^{2}$ in Fig. 4 are rescaled. The photoproduction data at high energies are from ZEUS and H1 [28. 29]; the other experiments are listed in Ref. 228]. The low energy data for electroproduction are from Ref. [30,31] and the high energy data from H1 [32]. The latter data correspond to $Q^{2}$ values slightly different from those of the low energy data and of our calculations. We see that all data are rather well described. The high $Q^{2}$ data indicate a preference for the fixed factorization scale of $4 m_{c}^{2}$ : the charm production at different (but not very large) $Q^{2}$ depends on the gluon distribution at a fixed scale. Therefore we will use this factorization scale also for diffractive charm production.

The deviation of the $\mathrm{H} 1$ results at $Q^{2}=12 \mathrm{GeV}^{2}$ from our theoretical prediction is partly explained by the fact that for our prediction we took the same $Q^{2}$ as for the low energy data, i.e. $Q^{2}=13.9 \mathrm{GeV}^{2}$. It means that our predictions are here slightly too small. Nevertheless, the discrepancy remains significant and is difficult to explain in view of the fact that for other values of $Q^{2}$ the agreement between theory and experiment is reasonable and that the cross sections have only a weak $Q^{2}$ dependence in this region. Except for these $\mathrm{H} 1$ points, our comparison confirms the photon-gluon fusion mechanism in combination with the CKMT gluon distribution at the factorization scale $4 m_{c}^{2}$.

In comparing our results to the data, it should be noted that the "resolved" diagrams of the type shown in Fig. 1b can contribute also for highly virtual photons. Our estimates indicate that this contribution at present energies is rather small - it is less than $10 \%$ of the main diagram of Fig. 1a. However in the same way as for real photons the contribution of Fig. 1b increases with energy faster than for Fig. 1a. Thus it will dominate at superhigh energies where it corresponds to charm production in the central rapidity region. 


\section{HARD DIFFRACTIVE SCATTERING AND CHARM PRODUCTION}

In deep inelastic scattering, there are events where particles are produced only in the fragmentation regions of the initial particles. We consider here the case that the proton remains intact, the so called "single diffractive scattering". This corresponds to a large "rapidity gap" between the diffractively produced states (with invariant mass $M_{X}$ ) and the recoil proton. This observation is readily explained in the Reggeon theory through the exchange of the Pomeron (Fig. 5a).

In this section, we discuss the diffractive cross section in the Pomeron exchange model [33] and specifically consider diffractive charm production. As in the total inclusive charm production discussed above, gluon fusion is again the dominant production mechanism for charm. Therefore, diffractive charm production is sensitive to the gluon distribution in the Pomeron, which is believed to consist mainly of gluons. This should be compared to the analogous inclusive process, where mainly the gluon component of the proton is probed. We will use the CKMT approach to obtain the parton distributions in the Pomeron and compare its results to two other models for the Pomeron parton distributions.

The differential production cross section can be written as

$$
\frac{d^{4} \sigma_{\mathrm{DIF}}}{d x d Q^{2} d x_{P} d t}=\frac{4 \pi \alpha_{E M}^{2}}{x Q^{4}}\left\{1-y+\frac{2 y^{2}}{2\left[1+R_{\mathrm{DIF}}\left(x, Q^{2}, x_{P}, t\right)\right]}\right\} F_{2}^{D}\left(x, Q^{2}, x_{P}, t\right)
$$

where we have introduced a diffractive structure function $F_{2}^{D}\left(x, Q^{2}, x_{P}, t\right)$. Here $x, y$ and $Q^{2}$ are the usual DIS variables and $t$ is the squared momentum transferred to the proton, $t \equiv k^{2}=\left(p-p^{\prime}\right)^{2}$. The variable $x_{P}$ is defined by

$$
x_{P}=\frac{q \cdot k}{q \cdot p} \simeq \frac{M_{X}^{2}+Q^{2}}{W^{2}+Q^{2}}
$$

with $M_{X}^{2}=(q+k)^{2}$ the squared invariant mass of the diffractively produced particles and $W^{2}=s=(q+p)^{2}$, the squared CMS energy of the photon-proton system. For the mechanism shown in Fig. $5 \mathrm{a}$, the variable $x_{P}$ can be interpreted as the fraction of the proton momentum carried by the Pomeron. The function $R_{\mathrm{DIF}}$ in Eq.(10) is the ratio of the longitudinal to transverse part of the cross section. Integrating $F_{2}^{D}\left(x, Q^{2}, x_{P}, t\right)$ over $x_{P}$ and $t$ we obtain the diffractive contribution to the total deep inelastic structure function $F_{2}\left(x, Q^{2}\right)$.

For the exchange of the Pomeron pole, the diffractive structure function $F_{2}^{D}\left(x, Q^{2}, x_{P}, t\right)$ can be factorized into two parts,

$$
F_{2}^{D}\left(x, Q^{2}, x_{P}, t\right)=f\left(x_{P}, t\right) F_{P}\left(\beta, Q^{2}, t\right)
$$

where the variable $\beta=Q^{2} /\left(M_{X}^{2}+Q^{2}\right)=x / x_{P}$ plays the same role as the Bjorken variable $x$ has in DIS: the Pomeron momentum fraction carried by the partons in the Pomeron. The first factor represents the Pomeron flux from the proton and can be written in the form

$$
f\left(x_{P}, t\right)=\frac{\left(g_{p p}^{P}(t)\right)^{2}}{16 \pi} x_{P}^{1-2 \alpha_{P}(t)}
$$

where $g_{p p}^{P}$ denotes the Pomeron-proton coupling. The value of $\alpha_{P}(t)$ in this flux factor should be taken at some effective virtuality scale, $Q_{e f f}^{2}$, since in the model of Ref. [9] one has

$$
\alpha_{P}(t)=1+\Delta\left(Q_{e f f}^{2}\right)+\alpha_{P}^{\prime} t
$$

where $\Delta$ is given by Eq.(7). The scale $Q_{\text {eff }}^{2}$ is a priori not known, but it was argued in Ref. "4 that it should be low, since a hard scale from the top part of Fig. 5b does not get through to the lower part of 
the diagram. From theoretical point of view, values of $\Delta\left(Q_{\text {eff }}^{2}\right)=0.13$ to $\Delta\left(Q_{\text {eff }}^{2}\right)=0.24$ are possible, corresponding to the effective Pomeron intercept without eikonal-type corrections and the "bare" value, respectively. Both extremes are not excluded by experimental evidence [2,3]. The Pomeron slope has its usual value $\alpha_{P}^{\prime}=0.25 \mathrm{GeV}^{-2}$. The second function, the Pomeron structure function $F_{P}$, is proportional to

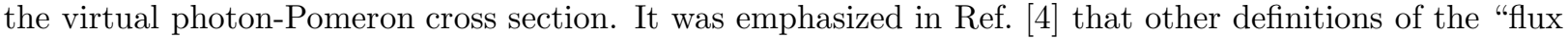
factor" are possible, e.g. differing from the one above by a constant. Therefore the normalization of the Pomeron structure function depends on the particular choice of the flux factor. For large values of $M_{X}$ or small values of $\beta$, the Mueller generalization of the optical theorem for inclusive cross sections can be used to represent the cross section given by Eq.(10) in terms of the triple-Reggeon diagram shown in Fig. 5b. The Reggeon exchange in the upper part of the diagram is dominated by the Pomeron and $f$ Regge poles. Since each term factorizes into a Reggeon propagator and a vertex, it is possible to obtain the structure function of the Pomeron from that of the deuteron $\llbracket$.

We will below consider the diffractive production involving light quarks and heavy quarks separately, i.e. we write the Pomeron structure function as

$$
F_{P}=F_{P}^{0}+F_{P}^{c \bar{c}} .
$$

For the contribution of light quarks to the structure function, we adopt the form for the deuteron structure function of Ref. [9], but with the couplings of the exchanged Reggeon to the deuteron replaced by the couplings to the Pomeron,

$$
F_{P}^{0}\left(\beta, Q^{2}, t\right)=e_{d}^{f} C_{f}^{d}\left(\beta, Q^{2}\right) \beta^{1-\alpha_{f}}(1-\beta)^{n\left(Q^{2}\right)-2}+e_{d}^{P} C_{P}^{d}\left(Q^{2}\right) \beta^{-\Delta\left(Q^{2}\right)}(1-\beta)^{n\left(Q^{2}\right)+2},
$$

with the ratios of the coupling constants

$$
e_{d}^{k}=\frac{r_{P P}^{k}(t)}{g_{d d}^{k}(0)}
$$

where $r_{P P}^{k}$ and $g_{d d}^{k}$ are the couplings of the Pomeron $(k=P)$ or the leading $f$ Regge pole $(k=f)$ to the Pomeron and to the deuteron, respectively. The values for the ratios $e_{d}^{k}$ can be estimated from soft diffraction data. In our calculations we will use $e_{d}^{P}=e_{d}^{f}=0.07$, which was shown in Ref. [5] to give a good description of the data on the diffractive structure function $F_{2}^{D}$. The behaviour of the structure function for $\beta \rightarrow 1$ is determined by the exponent $n\left(Q^{2}\right)$ which was obtained for the deuteron in Ref. [9] by using counting rules. For the Pomeron the exponent has to be adjusted accordingly since there is one parton spectator less.

From experiment it follows that the triple Reggeon couplings are only weakly dependent on $t$ and that for the Pomeron and the $f$ Regge pole exchange this dependence is approximately equal. It can then be incorporated in that of the flux factor in Eq.(12), i.e. in $g_{p p}^{P}$, which is then parametrized as $g_{p p}^{P}(t)=g_{p p}^{P}(0) \exp (C t)$ with $C=2.2 \mathrm{GeV}^{-2}$ and $g_{p p}^{P}(0)^{2}=23 \mathrm{mb}$. With this parametrization, the structure function of the Pomeron (or its parton distributions) do not have the $t$-dependence any more. The values of the parameters mentioned above have been all taken from Ref. [4]. We again use the parametrization at an initial scale of $Q_{0}^{2}=2 \mathrm{GeV}^{2}$ as starting point for $\mathrm{QCD}$ evolution to the desired $Q^{2}$ value. The contribution of the light quarks to the Pomeron structure function, $F_{P}$, is directly related to the corresponding singlet quark distribution of the Pomeron,

$$
F_{P}^{0}\left(\beta, Q^{2}\right)=\frac{2}{9} \Sigma_{P}\left(\beta, Q^{2}\right)
$$

with

$$
\Sigma_{P}\left(\beta, Q^{2}\right)=\sum_{i=u, d, s}\left[\beta q_{P}^{i}\left(\beta, Q^{2}\right)+\beta \bar{q}_{P}^{i}\left(\beta, Q^{2}\right)\right]
$$


This singlet distribution at $Q_{0}^{2}$ is shown in Fig. 6a.

We now discuss the charmed quark contribution to the diffractive Pomeron structure function, which is related to the analogous charm quark distribution,

$$
F_{P}^{c \bar{c}}\left(\beta, Q^{2}\right)=\frac{4}{9}\left[\beta c_{P}\left(\beta, Q^{2}\right)+\beta \bar{c}_{P}\left(\beta, Q^{2}\right)\right]
$$

Using photon-gluon fusion as the leading mechanism, the diffractive charm production can be calculated in the same way as for charm production in DIS in Section 2. This mechanism for diffractive production is shown in Fig. 7a. Thus while charm production in DIS was a probe of the gluon distribution in the proton, the diffractive production of charm now probes the gluon distribution in the Pomeron, which is our main point of interest here. In addition to the "sea" contribution in Fig. 7a, there is also a "valence" contribution from the Pomeron structure function. A typical example of such a contribution can be seen in Fig. $7 \mathrm{~b}$, which has been studied in Ref. [34]. The role of these additional mechanisms, which require further model assumptions, is not clear yet and had not been discussed in other papers on diffractive charm production. While the "sea" component is concentrated mostly at small values of $\beta$, it can be shown that the "valence" contribution has its maximum at $\beta \sim 0.5$ in Fig. $7 \mathrm{~b}$. Its value is smaller by a factor of order $1 / m_{c}^{2}$. In this region the "valence" contribution can therefore become comparable to the "sea" component.

In the CKMT approach, the gluon distribution of the Pomeron can for low $\beta$ be obtained from that of the proton (or deuteron), analogously to the Pomeron quark distribution discussed above. For a large parton momentum fraction, however, the analogy breaks down. The gluon distribution in the nucleon is determined at large $x$ by the gluon radiation originating from the quarks; in the Pomeron, which is believed to be mainly composed of gluons, they are a priori present. In Ref. [5] therefore the large $\beta$ behaviour was modified according to

$$
\beta g_{P}\left(\beta, Q^{2}\right)=e_{d}^{P} C_{g} \beta^{-\Delta\left(Q^{2}\right)}(1-\beta)^{n_{g}}
$$

where $n_{g}$ is a free parameter. The distributions we use are thus singular at $\beta=0$ due to the $\beta^{-\Delta}$ dependence dictated by the Pomeron exchange. The observed $Q^{2}$ dependence of the data indicate that the gluon distribution should be rather hard and thus $n_{g}$ negative, i.e. between 0 and -1 to yield a normalizable distribution. We use in the following two negative values of $n_{g}$, and therefore our gluon distribution is also singular for $\beta=1$. This can be seen in Fig.6b, where we show our distributions for a scale of $Q_{0}^{2}=2$ $\mathrm{GeV}^{2}$. For the actual calculation of diffractive charm production, we use QCD evolution to obtain the gluon distribution at the factorization scale of $4 m_{c}^{2}$.

Before showing our results for the diffractive production of light as well as charmed quarks, we first discuss the models of Refs. [6]8], where a similar description of the reaction involving the Pomeron structure function was used: model I of Gehrmann and Stirling (GS) [6] and model 3 of Golec Biernat and Kwiecinski $(\mathrm{GK})$ [7].

GK take the following singlet quark and gluon distributions, respectively, at $Q_{0}^{2}=4 \mathrm{GeV}^{2}$ :

$$
\Sigma_{P}(\beta)=0.069 K \beta^{0.44}(1-\beta)^{0.60}, \beta g_{P}(\beta)=1.16 K \beta^{5} \text {. }
$$

The constant $K$ relates the measured $t$-integrated diffractive structure function to the Pomeron structure function:

$$
\tilde{F}_{2}^{D}\left(x, Q^{2}, x_{P}\right) \equiv \int d t F_{2}^{D}\left(x, Q^{2}, x_{P}, t\right)=K\left(\frac{1}{x_{P}}\right)^{\Delta} F_{P}\left(\beta, Q^{2}\right)
$$

The value for $K$ depends on $x_{P}$ and is approximate 10 for the current experiments. The distributions used by GS at $Q_{0}^{2}=2 \mathrm{GeV}^{2}$ are:

$$
\Sigma_{P}(\beta)=1.02 \beta(1-\beta), \beta g_{P}(\beta)=4.92 \beta(1-\beta)
$$


In order to compare their quark and gluon distributions to ours, one has to take into account that different normalizations of the assumed Pomeron flux, $f\left(x_{P}, t\right)$, are used and they have to be converted to our conventions.

In Fig. 6a and 6b we compare the different model distributions for quarks and gluons in the Pomeron, all normalized according to our definition of the flux factor. We show the distributions as originally parametrized on their respective initial scales, $Q_{0}^{2}$. The quark singlet distribution, $\Sigma_{P}$, looks similar in the three models. Except for the behaviour of the CKMT parametrization at very low $\beta$, the singlet quark distributions show roughly the same qualitative features for all models, because they were fitted to diffractive production data for $\beta \geq 0.065$. Some differences with the CKMT model are due to the fact that the other models fit to all the diffractive data and thus also simulate doubly diffractive data with their fit, which account for about $30 \%$ of the cross section.

In contrast to the quark distributions the gluon distributions of the three models are dramatically different: the predicted distributions have different shapes. The GK parametrization is the "hardest" of the three types of distributions, remaining essentially zero for low beta and having its main strength near $\beta=1$. The distribution of GS is symmetric, vanishing at $\beta=0$ and $\beta=1$, and thus is peaked in the middle. The gluons in the CKMT model are distributed with nearly constant density for intermediate $\beta$ values; their distribution diverges at both ends. It becomes steeper at $\beta=1$ the closer $n_{g}$ gets near -1 . The very large values of $g_{P}(\beta)$ for intermediate values of $\beta$ in the GS and GK models are difficult to reconcile with the cross sections for jet production in diffractive hadronic interactions 35. The most direct test of all models is provided by the diffractive production of heavy quarks and we will therefore now look at diffractive charm production in DIS.

For the calculations, the gluon distributions are used at the common factorization scale of $4 m_{c}^{2}$. They are shown in Fig. 6d and can be seen to have rather different qualitative features than in Fig. 6b. The QCD evolution from the initial scale $Q_{0}^{2}$ to the higher scale shifts the gluon distributions towards lower momentum fractions. This can be most clearly seen for the GS model. All gluon distributions are now singular at $\beta=0$. For GS and GK this is due to the evolution, while CKMT already starts out singular at the origin due to the $\beta^{-\Delta}$ behaviour, which is only somewhat modified by QCD evolution. The opposite effect can be seen at $\beta=1$ : the originally singular distribution in the CKMT model becomes zero. For completeness, we also show an example for the effect of evolution on the light quark distribution in Fig. 6c. For GS and GK, the magnitude of the distribution increases for moderate values of $\beta$. Furthermore, again a slight shift towards small $\beta$ occurs. For CKMT, the QCD evolution makes the density becomes more flat and the change in magnitude is such that the distribution is lower than the others at intermediate $\beta$.

One has to be careful when considering the energy-momentum sum rule, i.e. the integral over $\beta$ of the sum of the gluon and quark distributions times $\beta$. As was pointed out before, the choice of the Pomeron flux factor fixes the normalization of the Pomeron structure function and therefore the value of the energymomentum sum rule. This sum rule needs not to be equal to 1 as was assumed in several publications. However, the value of this energy-momentum sum is preserved under QCD evolution. "virtual particle". This explains the changes in magnitude discussed above: in response to an overall growth in the quark density the gluon density decreases. At the factorization scale, the total gluon content of the Pomeron predicted by CMKT is $60 \%$ and about $80 \%$ for the other models.

The contribution of charm quarks to the Pomeron structure function, produced by the gluon-photon fusion mechanism is shown in Fig. 8 for the three gluonic distributions discussed above and for different values of $Q^{2}$. The results are obtained by carrying out a convolution analogous to electroproduction. To obtain $F_{P}^{c \bar{c}}$ at a given momentum fraction $\beta$ carried by the struck charmed quark, we have to integrate over gluon momentum fractions starting from a $Q^{2}$ dependent cut-off value that follows from the lower limit in Eq.(11). Both the CKMT and GS result increase continously as $\beta$ decreases from this cut-off. The GK result has a maximum about halfway which originates from its relatively hard gluon distribution. As the virtuality of the photon increases, the cut-off moves to larger values, but the general shape of the curves doesn't change. For a $Q^{2}$ value as high as $500 \mathrm{GeV}^{2}$, the factorization scale we have chosen may not be appropriate anymore and a $Q^{2}$ dependent value might be better. In general, the shown predictions are sufficiently different such that future experiments for charm production in hard diffractive scattering should be able to discriminate between them. 
To see how significant the charm production contributes to the total diffractive cross section, we also show in Fig. 8 the full $F_{P}$. Adding these charm quark contributions results in a (small) $Q^{2}$ dependent violation of the sum rule discussed above. The fraction of the total structure function provided by the charm is in all cases most important for small $\beta$ and increases with $Q^{2}$. A measurement of the total diffractive cross section is of course a test of the different parton density input. contributions,

The exponent $n_{g}$, which determines the "hardness" of the CKMT gluon distribution, is a free parameter. To see how sensitive the results are to its value, we compare in Fig. 9 results for $F_{P}$ and $F_{P}^{c \bar{c}}$ for $n_{g}=-0.5$ and -0.9 at the same $Q^{2}$ values as in Fig. 8 . The charm part, $F_{P}^{c \bar{c}}$, is most directly sensitive to the gluon distribution. The shape of the curves does not change much. However, as $F_{P}^{c \bar{c}}$ receives its contribution from gluons with high momentum fraction $\beta$ due to the presence of the cut-off in the convolution Eq.(1i), the harder distribution, $n_{g}=-0.9$, yields a significantly larger prediction. This is most pronounced at high $\beta$ and similar for all values of $Q^{2}$. For the total $F_{P}$, the more singular gluon distribution results in an increase, in particular at larger $Q^{2}$. The exponent $n_{g}$ enters on the one hand through the charm contribution discussed above. On the other hand, it also influences the light quark contribution through the QCD evolution. The curves clearly show that the hardness of the gluon distribution is relevant for the total diffractive cross section.

It is interesting to see the contribution of the diffractive production to the total DIS structure function, $F_{2}\left(x, Q^{2}\right)$. To compare to the results in Section 2, we convert this into the virtual photon cross section, $\sigma\left(W, Q^{2}\right)$. For this purpose, we must integrate the diffractive contribution in Eq.(12) over $x_{P}$ and $t$. For $x_{P}$ we integrate up to 0.1 , which defines our lower limit for the rapidity gap. We use for the Pomeron intercept in the flux factor a value $\alpha_{P}(0)=1.13$ as in Ref. [5]. We show in Fig. 10 these diffractive contributions at the same $Q^{2}$ values as for the total in Fig. 4. They increase rapidly from their threshold and then reach an approximately powerlike $W$-dependence. Comparison of Figs. 4 and 10 shows that the diffractive production is for large energies an order of magnitude smaller than the total. For large energies, the result for the harder gluon distribution is about a factor 1.5 higher.

\section{BEAUTY PRODUCTION}

We now repeat some of the above calculations for the production of beauty quarks. Due to the larger mass, this of course involves a different factorization scale and thus probes the gluon distribution at a much higher scale; we take it as $4 m_{b}^{2}$. The cross section can be calculated using the same photon-gluon fusion mechanism and the approach discussed above. The only difference is the mass of the b-quark, which we take as $m_{b}=4.7 \mathrm{GeV}$. It can be found using the mass of the charm quark from the following relation

$$
m_{b}-m_{c}=\bar{m}_{B}-\bar{m}_{D}
$$

which is valid in QCD up to small corrections $\sim 1 / m_{Q}$ [36]. The quantity $\bar{m}_{B} \equiv\left(m_{B}+3 m_{B^{*}} / 4\right)$ in Eq.(25) denotes the center of gravity value for the lowest mesonic state (the same holds for $\bar{m}_{D}$ ).

Predictions for the cross section for beauty production by real and virtual photons are shown in Fig. 11. The threshold is higher than for charm production, but the general shape of the cross section is similar. The magnitude is typically two orders of magnitude lower, which is due to the larger mass and the smaller charge. The contribution of diffractive beauty production to $F_{P}\left(\beta, Q^{2}\right)$ is shown in Fig. 12 . The $\beta$ value for which the result becomes zero has decreased due to the larger quark mass. The shape of the curves, their dependence on $Q^{2}$ and the hardness of the gluon function are similar as for charm production, Fig. 9. The contribution to the structure function $F_{2}\left(x, Q^{2}\right)$ is shown in Fig. 13. The threshold energy is now higher, but for the rest a similar behaviour to charm production can be seen: as in Fig. 10, the diffractive contribution is again an order of magnitude smaller than the total DIS beauty production and the sensitivity to the hardness of the gluon distribution looks the same. In general, these cross sections are much smaller than in the case of charm and large statistics is needed to observe them experimentally. 


\section{SUMMARY}

The main goal of this paper was to probe the gluon distribution in the Pomeron by means of diffractive heavy quark (charm, beauty) production. Given the present uncertainty in the data as well as the high sensitivity of perturbative QCD calculations to the heavy quark mass, we have here pursued a simple phenomenological approach to obtain a description of the main features of heavy quark production. First we showed that the data for total open charm photo- and electroproduction could be well described by using the gluon distribution of the proton predicted by the CKMT model, together with the photon-gluon fusion mechanism. From this comparison to the data we extracted a value of $m_{c}=1.4 \mathrm{GeV}$ for the charm quark mass parameter and confirmed the finding of Ref. 15] that $4 m_{c}^{2}$ is a good factorization scale. Having established this basis, we proceeded to diffractive charm production. We obtained results with gluon distributions of different models and compared their contribution to the total diffractive structure function of the Pomeron. For the CKMT model, we investigated how the results depend on the high momentum behaviour of the gluon distribution. A similar study was made for beauty production.

In conclusion we found that different models for the gluonic content of the Pomeron lead to sizeable differences for diffractive charm production which experiments, such as the future high statistics experiments at HERA [37], should be able to distinguish. For beauty production, similar features were found, but the cross sections are much smaller and thus more difficult to measure.

\section{ACKNOWLEDGEMENTS}

The work of L.H. and J.K. is part of the research program of the Foundation for Fundamental Research of Matter (FOM) and the National Organization for Scientific Research (NWO). The collaboration with ITEP is supported in part by a grant from NWO and by grant 93-79 of INTAS. A.K. also acknowledges support from grant 96-02-19184 of RFFI.

\section{APPENDIX}

For the QCD evolution equation one needs the quark distributions at an initial scale $Q_{0}^{2}$. Up to $Q^{2}=5 \mathrm{GeV}^{2}$ the total valence and sea quark distribution in LO can directly be extracted from $F_{2}\left(x, Q^{2}\right)$ as parametrized by the CKMT model in Ref. [9]:

$$
\begin{aligned}
& F_{2}^{v a l}\left(x, Q^{2}\right) \equiv \frac{4}{9} u^{v}\left(x, Q^{2}\right)+\frac{1}{9} d^{v}\left(x, Q^{2}\right)=C_{f}\left(x, Q^{2}\right) x^{1-\alpha_{f}}(1-x)^{n\left(Q^{2}\right)} \\
& F_{2}^{s e a}\left(x, Q^{2}\right) \equiv \frac{8}{9} u^{s}\left(x, Q^{2}\right)+\frac{2}{9} d^{s}\left(x, Q^{2}\right)+\frac{2}{9} s^{s}\left(x, Q^{2}\right)=C_{P}\left(Q^{2}\right) x^{-\Delta\left(Q^{2}\right)}(1-x)^{n\left(Q^{2}\right)+4}
\end{aligned}
$$

with the functions

$$
\begin{array}{ll}
C_{f}\left(x, Q^{2}\right)=B(x)\left(\frac{Q^{2}}{Q^{2}+b}\right)^{\alpha_{R}} \quad, & C_{P}\left(Q^{2}\right)=A\left(\frac{Q^{2}}{Q^{2}+a}\right)^{1+\Delta\left(Q^{2}\right)} \\
\Delta\left(Q^{2}\right)=\Delta(0)\left(1+\frac{d_{0} Q^{2}}{Q^{2}+d_{1}}\right) \quad, \quad n\left(Q^{2}\right)=\frac{3}{2}\left(1+\frac{Q^{2}}{Q^{2}+c}\right)
\end{array}
$$

This parametrization has been constructed in such a way that for $x \sim 1$ it is in accordance with the dual parton model at low $Q^{2}$ and with dimensional counting rules at very large $Q^{2}$. The low $x$ behaviour is readily explained in terms of Reggeon exchanges; the secondary Regge trajectory with intercept $\alpha_{f}$, corresponding to $\left(f, A_{2}\right)$-exchanges, determines the small $x$ distributions for the valence quarks, while the "effective" Pomeron exchange determines them for the sea quarks (and the gluon). The exponents in $C_{f}$ and $C_{P}$ are chosen such that the photolimit $\left(Q^{2}, x \rightarrow 0\right)$ is finite. 
The function $B$ was written as the sum of the $u$ and $d$ quark contribution, $B=B_{u}+B_{d}$, and is different for the proton and the deuteron:

$$
\begin{array}{lll}
B_{u}=\frac{4}{9} C_{u} & , B_{d}=\frac{1}{9} C_{d}(1-x) & \text { for the proton } \\
B_{u}=\frac{2}{9}\left[C_{u}+C_{d}(1-x)\right] & , B_{d}=\frac{1}{18}\left[C_{u}+C_{d}(1-x)\right] & \text { for the deuteron }
\end{array}
$$

where the constants $C_{u}$ and $C_{d}$ were obtained from the condition that the integrals of the valence distributions over $x$ should give the correct number of valence up and down quarks in the proton. Here the constants for the deuteron are related to those of the proton through isospin symmetry. For the sea quarks the simple assumption is used that $u^{s}=d^{s}$ and $s^{s}=0.5 u^{s}$, in reasonable accordance with the results from $\nu N$ interactions.

Also the gluon distribution for low $x$ is determined by the Pomeron exchange and is thus proportional to the sea quark distributions. However, since sea quarks are produced mostly by gluons, the distribution of gluons will be harder than the one of the sea quarks. This leads to the gluon distribution in Eq.(6):

$$
x g\left(x, Q^{2}\right)=G \frac{F_{2}^{s e a}\left(x, Q^{2}\right)}{1-x}=C_{g}\left(x, Q^{2}\right) x^{-\Delta\left(Q^{2}\right)}(1-x)^{n\left(Q^{2}\right)+3},
$$

where the proportionality factor $G$ was obtained from the momentum sum rule at $Q^{2}=2 \mathrm{GeV}^{2}$.

The parameter $d_{0}$ in the effective Pomeron intercept was originally 9 put equal to 2 in order to have for the bare Pomeron a value of $\Delta_{\text {bare }}$ that is 3 times larger than $\Delta(0)$, in agreement with analyses of hadronic interactions [27]. The seven remaining free parameters of the model were fitted in Ref. [9] to the data on total $\gamma p$ interaction cross section and to the proton structure function data of NMC. They are given by

$$
\begin{aligned}
& a=0.2631 \quad, \quad b=0.6452 \quad, \quad c=3.5489 \quad, \quad d_{1}=1.1170 \quad, \\
& A=0.1502 \quad, \quad \Delta(0)=0.07684 \quad, \quad \alpha_{f}=0.4150 \text {. }
\end{aligned}
$$

Here the parameters $a, b, c$ and $d_{1}$ are given in units of $\mathrm{GeV}^{2}$. With these values for the free parameters one finds explicitly for the coefficients:

$$
C_{u}=2.714, C_{d}=1.618, G=12.63
$$

However, using new HERA data in the region of $Q^{2}<2 \mathrm{GeV}^{2}$ [38,39] it is possible to determine the parameters $d_{0}$ and $d_{1}$ with better accuracy: $d_{0}=2.2, d_{1}=0.6 \mathrm{GeV}^{2}$.

[1] A.B. Kaidalov, Surveys in High Energy Physics, 9 (1996) 143

[2] M. Derrick et al. (ZEUS), Phys. Lett. B 315 (1993) 481; M. Derrick et al (ZEUS), Z.Phys. C 70 (1996) 391

[3] T. Ahmed et al. (H1), Nucl. Phys. B 429 (1994) 477; T. Ahmed et al. (H1), Phys. Lett. B 348 (1995) 681

[4] A. Capella, A. Kaidalov, C. Merino and J. Tran Thanh Van, Phys. Lett. B 343 (1995) 403.

[5] A. Capella, A. Kaidalov, D. Pertermann and J. Tran Thanh Van, LPTHE Orsay 95-33

[6] T. Gehrmann and W.J. Stirling, Z.Phys. C 70 (1995) 227

[7] K. Golec-Biernat and J. Kwiecinski, Phys. Lett. B 353 (1995) 329; K. Golec-Biernat, DTP $96-32$ (1996)

[8] J.B. Dainton (H1 Collaboration), DESY-95-228

[9] A. Capella, A. Kaidalov, C. Merino and J. Tran Thanh Van, Phys. Lett. B 337358 (1994)

[10] L.P.A. Haakman, A. Kaidalov and J.H.Koch, Phys. Lett. B 365411 (1996)

[11] J.C. Collins and W.-K. Tung, Nucl. Phys. B 278 (1986) 934

[12] M. Gluck, E. Reya and A. Vogt, Z.Phys. C 53 (1992) 127.

[13] A.D. Martin, R.G. Roberts and W.J. Stirling, Phys. Rev. D 50 (1994) 6734 
[14] H.L. Lai et al. (CTEQ Collaboration), Phys. Rev. D 51 (1995) 4763

[15] M. Gluck, E. Reya, M. Stratmann, Nucl. Phys. B 422 (1994) 37

[16] R.K. Ellis and P. Nason, Nucl. Phys. B 312 (1989) 551

[17] J. Smith and W.L. van Neerven, Nucl. Phys. B 374 (1992) 225

[18] E. Laenen, S. Riemersma, J. Smith and W.L. van Neerven, Nucl. Phys. B 392 (1993) 162

[19] E. Laenen, hep-ph 9609351

[20] M. Buza, Y. Matiounine, J. Smith and W.L. van Neerven, Preprint NIKHEF-96-027

[21] M.L. Mangano, P. Nason and G. Ridolfi, Nucl. Phys. B 373 (1992) 295; S. Frixione, M. Mangano, P. Nason and G. Ridolfi, Nucl Phys. B 412 (1994) 225

[22] S. Frixione, M.L. Mangano, P. Nason, G. Ridolfi, Preprint CERN-TH.752794 (1994)

[23] M.A. Shifman, A.I. Vainstein and V.I. Zakharov, Nucl. Phys. B 136 (1978) 157

[24] E. Witten, Nucl. Phys. B 104 (1976) 445

[25] M. Derrick et al. (ZEUS), Phys. Lett. B 293 465; M. Derrick et al. (ZEUS), Phys.Lett. B 316 (1993) 412

[26] T. Ahmed et al. (H1), Phys. Lett. B 299 (1992) 85; I. Abt et al. (H1), Nucl. Phys. B 407 (1993) 515

[27] A.B. Kaidalov, L.A. Ponomarev and K.A. Ter-Martirosyan, Sov. J. Nucl. Phys. 44 (1986) 468

[28] M. Derrick et al. (Zeus), Phys. Lett. B 349 (1995) 225

[29] S. Aid et al. (H1), Nucl. Phys. B 472 (1996) 32

[30] J.J. Aubert et. al. (EMC), Nucl. Phys. B 213 (1983) 31

[31] A. Donnachie and P.V. Landshoff, Z.Phys. C 61 (1994) 139

[32] Adloff et al. (H1), DESY-96-138 (1996)

[33] G. Ingelman and P.E. Schlein, Phys. Lett. B 152 (1985) 256 ; G. Ingelman and K. Prytz, Z.Phys. C58 (1993) 285

[34] N.N. Nikolaev and B.G. Zakharov, Z.Phys. C 53 (1992) 331 ; M. Genovese, N.N. Nikolaev and B.G. Zakharov, Phys. Lett. B 378 1992) 347

[35] K. Goulianos, Preprint RU-95-E-26

[36] I.Bigi, M. Shifman, N. Uraltsev and A. Vainshtein, Phys. Lett. B 328 (1994) 431

[37] R. Eichler and S. Frixione, Preprint ETH-TH/96-34

[38] M. Derrick et al. (ZEUS), Z.Phys. C 69 (1996) 607

[39] G. Bernardi (H1), Preprint LPNHE-96-02 


\section{FIGURE CAPTIONS}

Fig. 1 a. Photon-gluon fusion diagram.

b. Charm production by the gluon-gluon fusion mechanism ("resolved" contribution).

Fig. 2 Charm photoproduction cross section predicted by the CKMT model. Solid lines: direct contribution for different values of the charm mass; from top to bottom $m_{c}=1.3,1.4,1.5,1.6 \mathrm{GeV}$. Lower dashed line: resolved contribution with $m_{c}=1.4 \mathrm{GeV}$ obtained with the gluon distribution in the photon according to the CKMT model. Upper dashed line: the sum of the direct and resolved contribution $\left(m_{c}=1.4 \mathrm{GeV}\right)$.

Fig. 3 a. Charm contribution to the proton structure function $F_{2}\left(x, Q^{2}\right)$ in the CKMT approach as function of $Q^{2}$ for different $x$; from top to bottom: $x=0.0001,0.001,0.01,0.1$. Solid lines are obtained by using the gluon fusion process below $Q^{2}=50 \mathrm{GeV}^{2}$ and massless QCD evolution for $Q^{2}>50 \mathrm{GeV}^{2}$. Dashed lines: charm contribution by using the gluon fusion model for all $Q^{2}$. Factorization scale is $\mu_{f}^{2}=4 m_{c}^{2}$.

b. Logarithmic derivative of $F_{2}^{c \bar{c}}$ obtained from Fig. 3a for $x=0.1$ (dot-dashed), $x=0.01$ (dotted), $x=0.001$ (dashed) and $x=0.0001$ (solid line)

c. Same figure as a., but with factorization scale $\mu_{f}^{2}=4 m_{c}^{2}+Q^{2}$.

d. Same figure as b., but with factorization scale $\mu_{f}^{2}=4 m_{c}^{2}+Q^{2}$.

Fig. 4 Comparison of the charm photo-and electroproduction cross sections predicted in this paper with experiment for different $Q^{2}$; from top to bottom $Q^{2}=0,1.39,2.47,4.39,7.81,13.9,24.7,43.9,78.1 \mathrm{GeV}^{2}$. Solid lines: $\mu_{f}^{2}=4 m_{c}^{2}$. Dashed lines: $\mu_{f}^{2}=4 m_{c}^{2}+Q^{2}$. All curves are rescaled with powers of 10 ; the $\mathrm{k}$-th curve from the top is rescaled with a factor $10^{-k}$. The data are from [28, 30, 32].

Fig. 5 a. Diffractive dissociaton of the photon in photon-proton scattering due to the Pomeron exchange. b. Triple-regge diagram for hard diffractive scattering in the small $\beta$ region.

Fig. 6 a. Different parametrizations for the light quark distribution of the Pomeron, $\Sigma_{P}(\beta)$, at initial scales $Q_{0}^{2}$. Solid line: this paper with $n_{g}=-0.5$. Dotted line: this paper with $n_{g}=-0.9$. Dot-dashed line: GS [6]. Dashed line: GK [7].

b. Different gluonic distributions in the Pomeron at initial scales $Q_{0}^{2}$. Solid line: this paper with $n_{g}=-0.5$. Dotted line: this paper with $n_{g}=-0.9$. Dot-dashed line: GS [6]. Dashed line: GK [7].

c. Predictions for the light quark singlet distributions at the factorization scale by different models. Solid line: this paper with $n_{g}=-0.5$. Dotted line: this paper with $n_{g}=-0.9$. Dot-dashed line: GS [6]. Dashed line: GK [7].

d. Predictions for gluon distributions at the factorization scale by different models. Solid line: this paper with $n_{g}=-0.5$. Dotted line: this paper with $n_{g}=-0.9$. Dot-dashed line: GS [6]. Dashed line: GK [7].

Fig. 7 a. Pomeron "sea" charm contribution.

b. Typical diagram that contributes to the "valence" charm component of the Pomeron.

Fig. 8 Pomeron structure function (upper curves) and its charm contribution (lower curves) predicted by different models as function of $\beta$ at different $Q^{2}$. Solid line: this paper with $n_{g}=-0.5$. Dot-dashed line: GS [6]. Dashed line: GK [7].

a. $Q^{2}=10 \mathrm{GeV}^{2}$.

b. $Q^{2}=100 \mathrm{GeV}^{2}$.

c. $Q^{2}=500 \mathrm{GeV}^{2}$.

Fig. 9 CKMT predictions with different $n_{g}$ for the Pomeron structure function (upper curves) and its charm contribution (lower curves). Solid lines: $n_{g}=-0.5$. Dotted lines with $n_{g}=-0.9$. For every set the $Q^{2}$-values are from top to bottom: $Q^{2}=500,100,10 \mathrm{GeV}^{2}$.

Fig. 10 Diffractive charm contribution to the total charm production cross section for different values of $Q^{2}$; from top to bottom $Q^{2}=0,1.39,2.47,4.39,7.81,13.9,24.7,43.9,78.1 \mathrm{GeV}^{2}$. Solid lines: $n_{g}=-0.5$. Dotted lines: $n_{g}=-0.9$.

Fig. 11 Cross sections for photo-and electroproduction of beauty predicted by this paper for different values of $Q^{2}$; from top to bottom $Q^{2}=0,1.39,2.47,4.39,7.81,13.9,24.7,43.9,78.1 \mathrm{GeV}^{2}$.

Fig. 12 Beauty contribution to the Pomeron structure function predicted by this paper at different values of $Q^{2}$ : from top to bottom $Q^{2}=500,100,10 \mathrm{GeV}^{2}$. Solid lines: $n_{g}=-0.5$. Dotted lines: $n_{g}=-0.9$.

Fig. 13 Diffractive beauty contribution to the total beauty production cross section predicted by this paper for different values of $Q^{2}$; from top to bottom $Q^{2}=0,1.39,2.47,4.39,7.81,13.9,24.7,43.9,78.1 \mathrm{GeV}^{2}$. Solid lines: $n_{g}=-0.5$. Dotted lines: $n_{g}=-0.9$. 


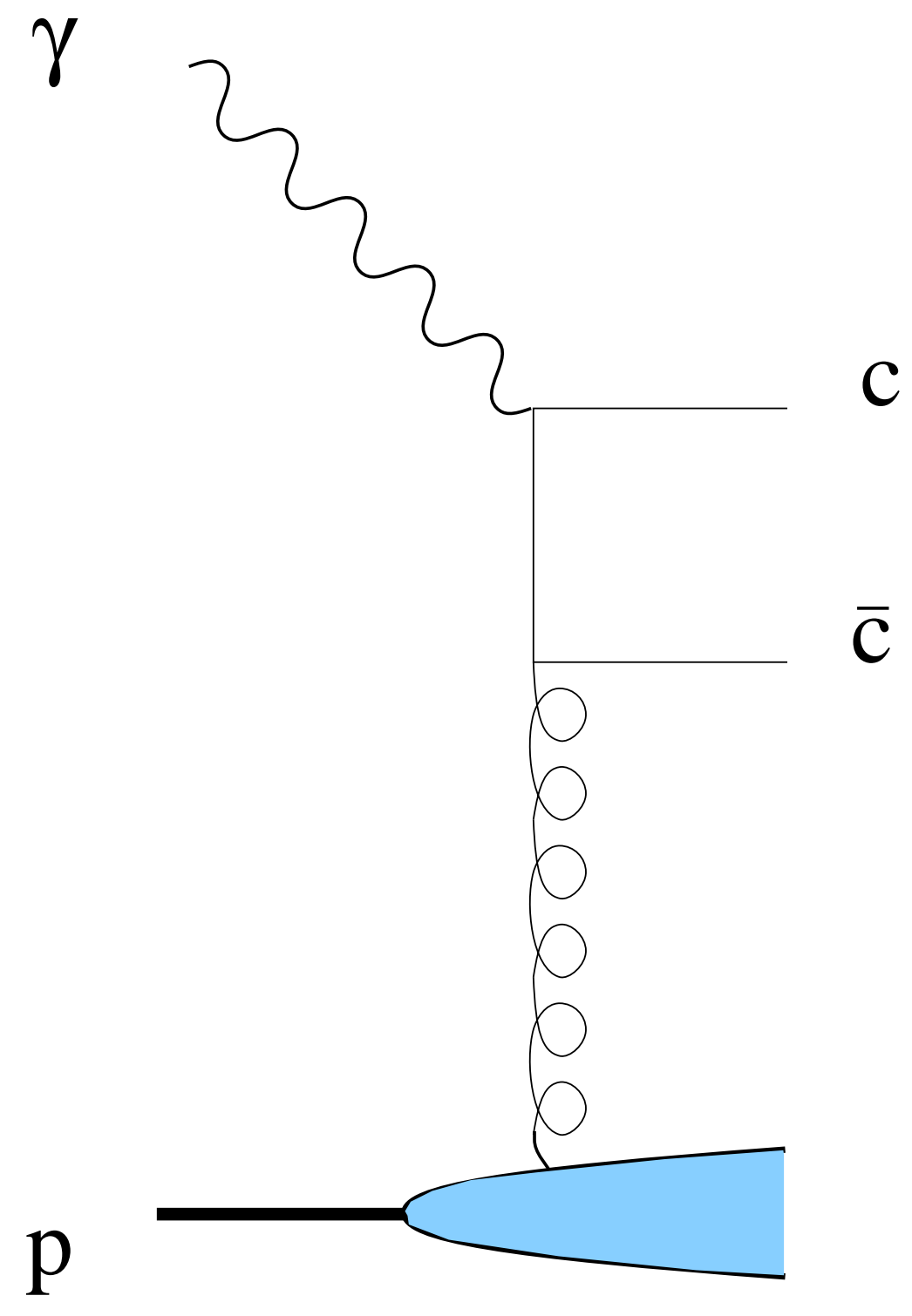

Figure 1a 

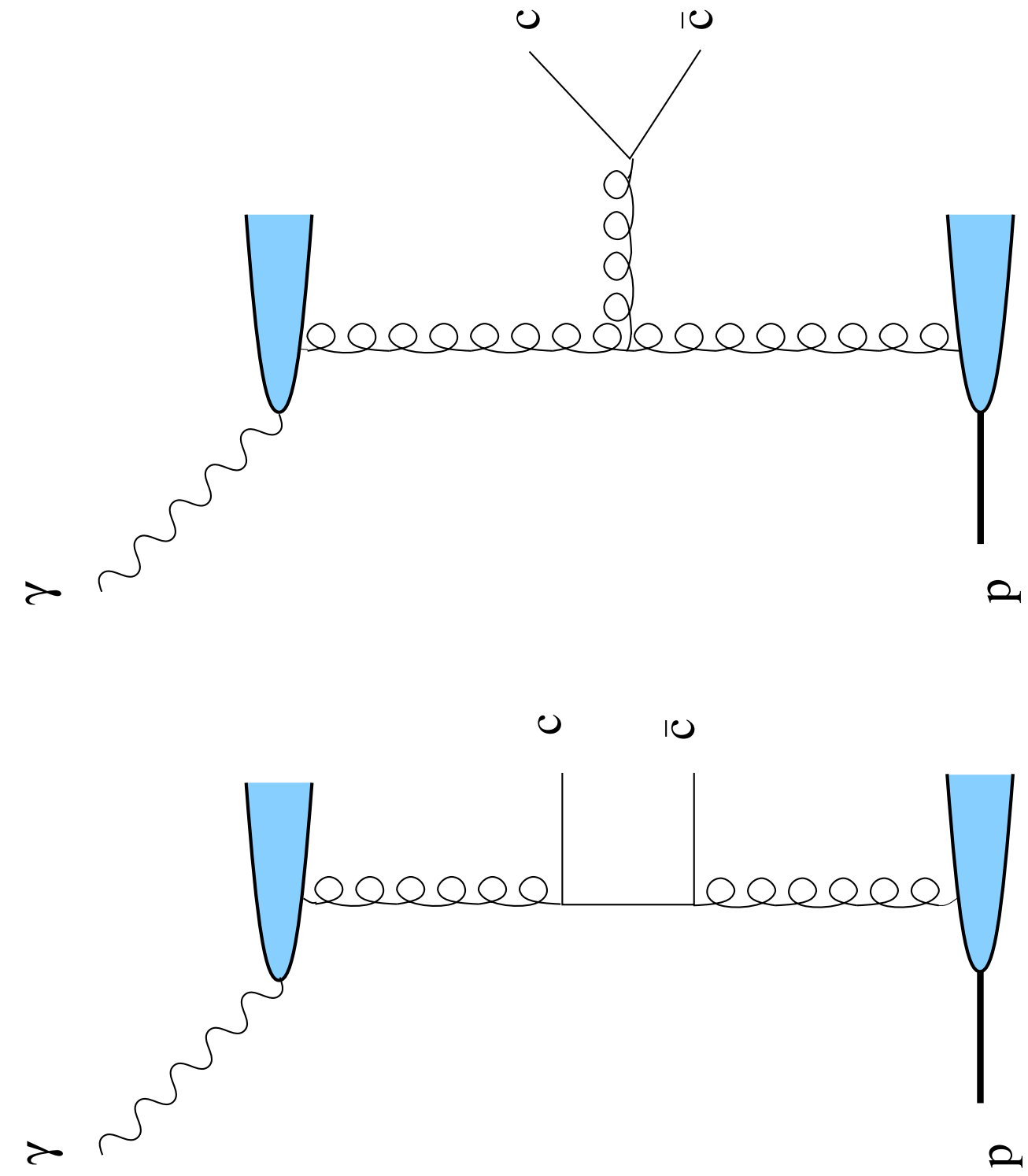

Figure 1b 


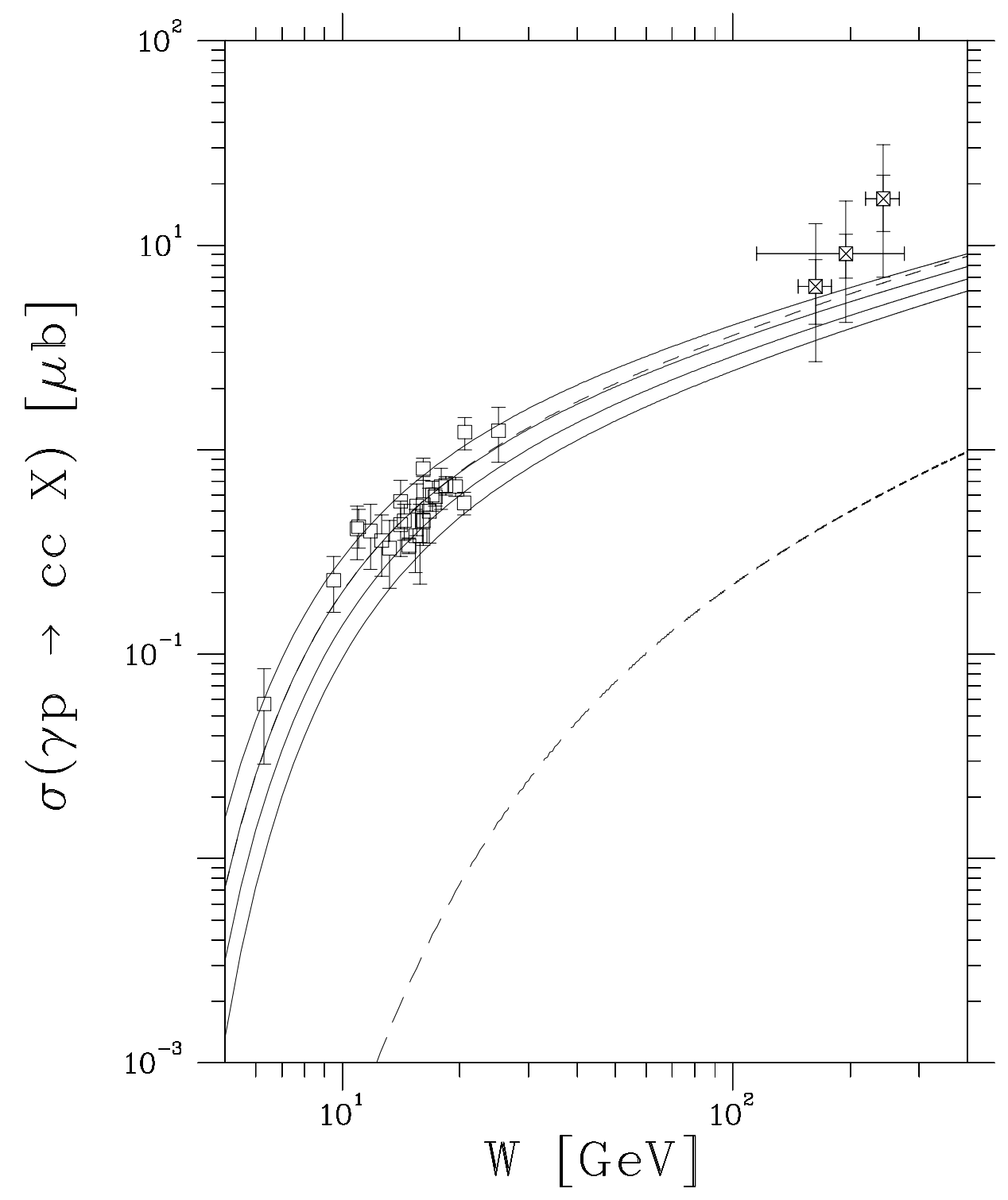

Figure 2 


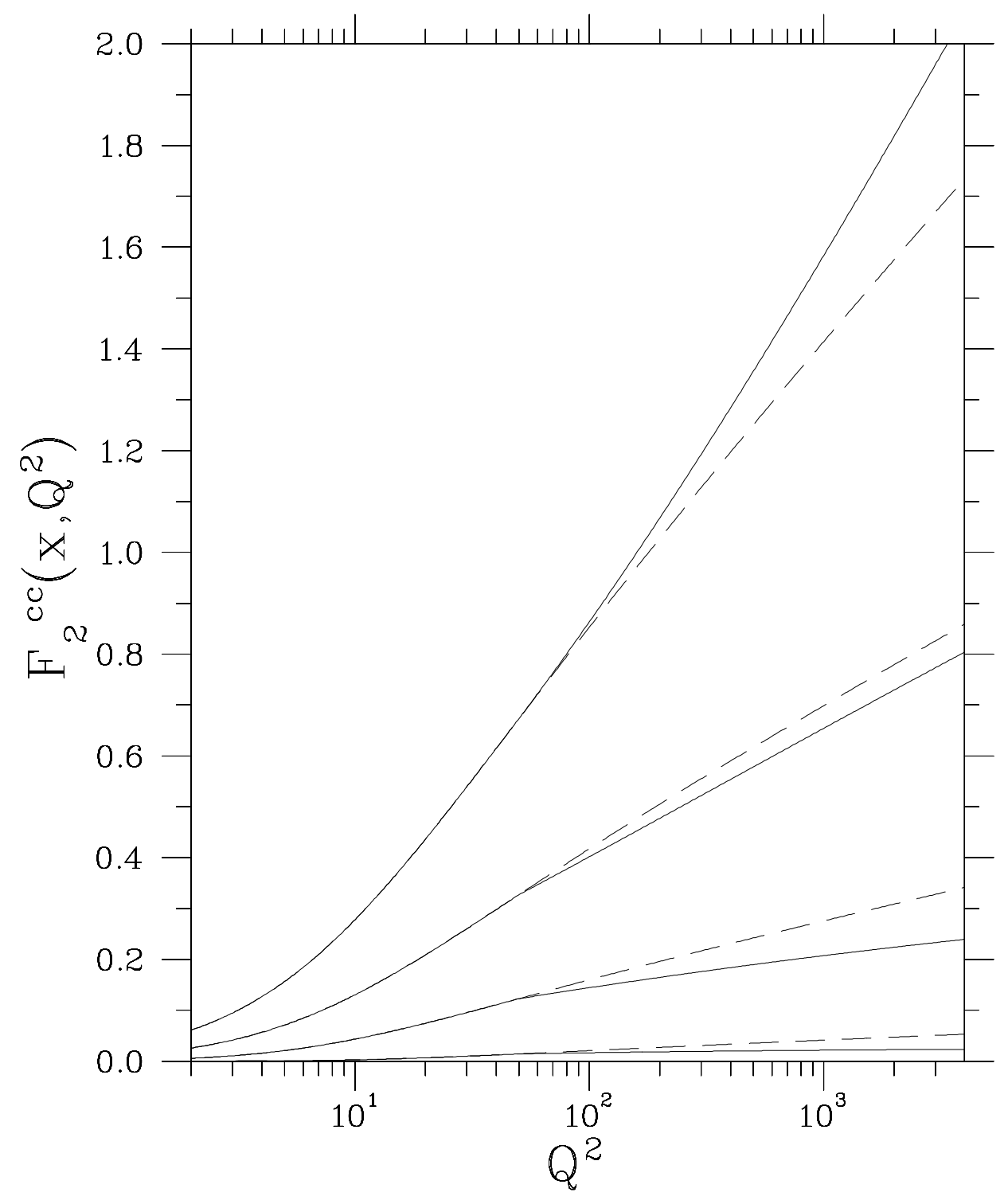

Figure 3a 


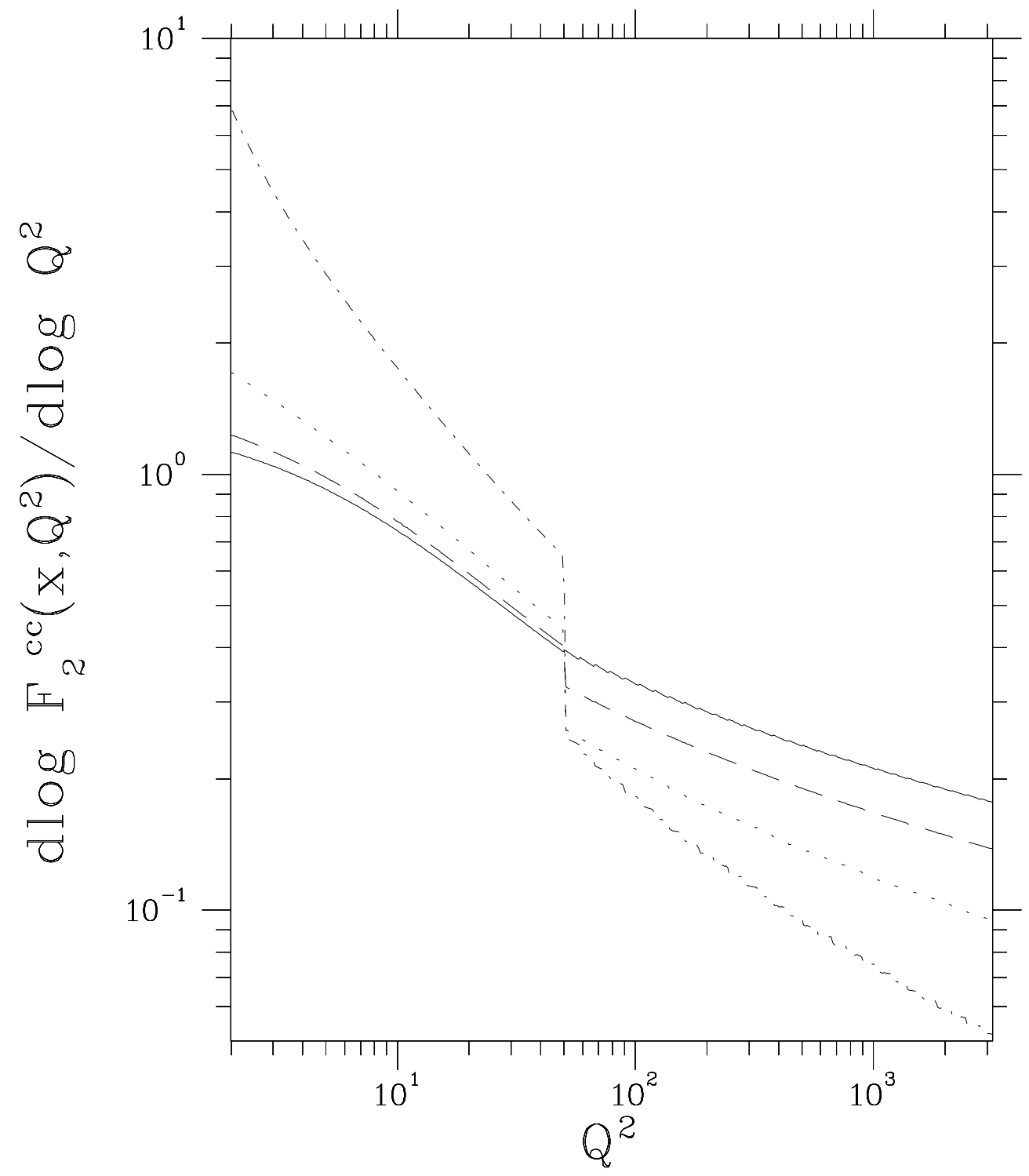

Figure $3 b$ 


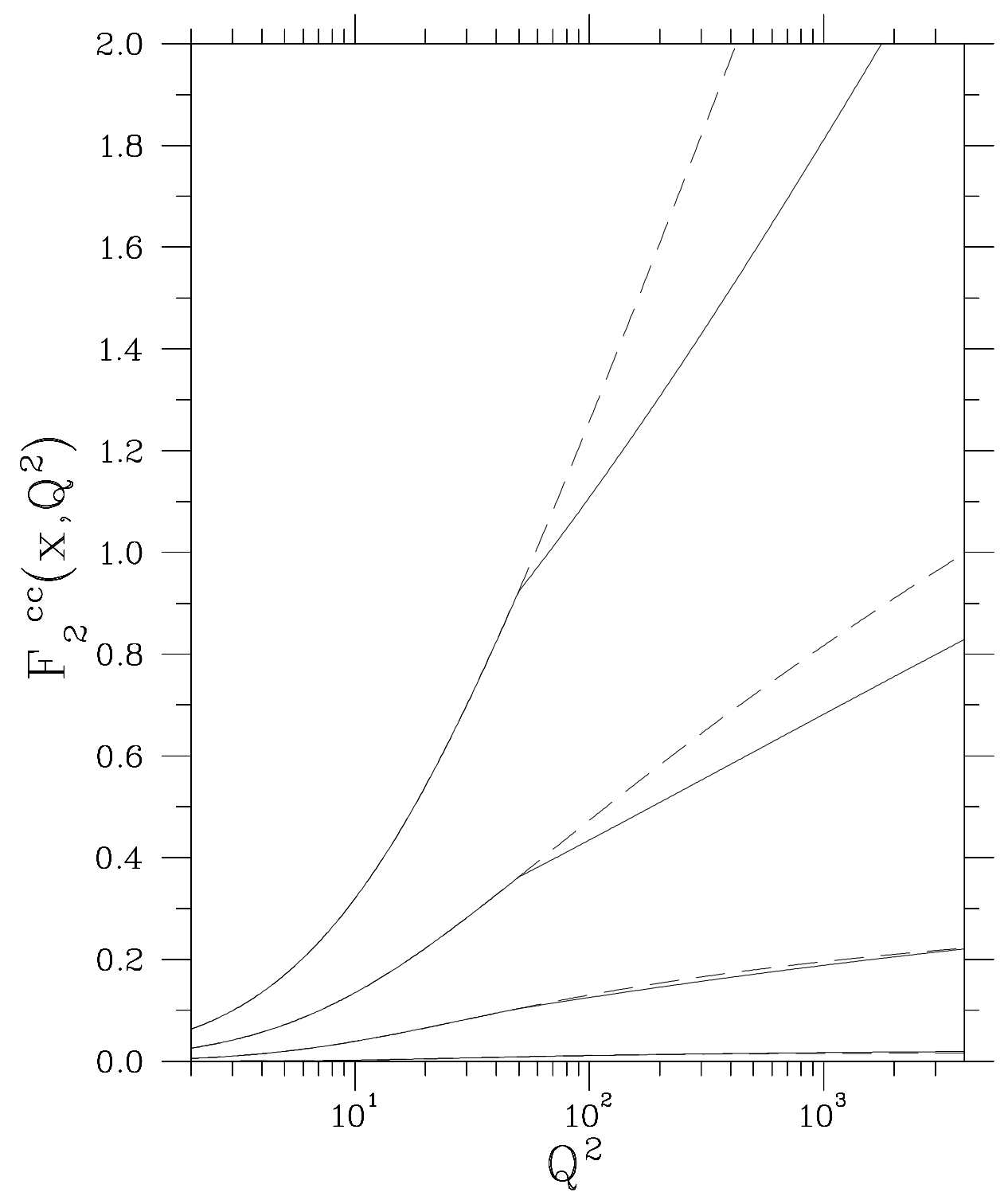

Figure 3c 


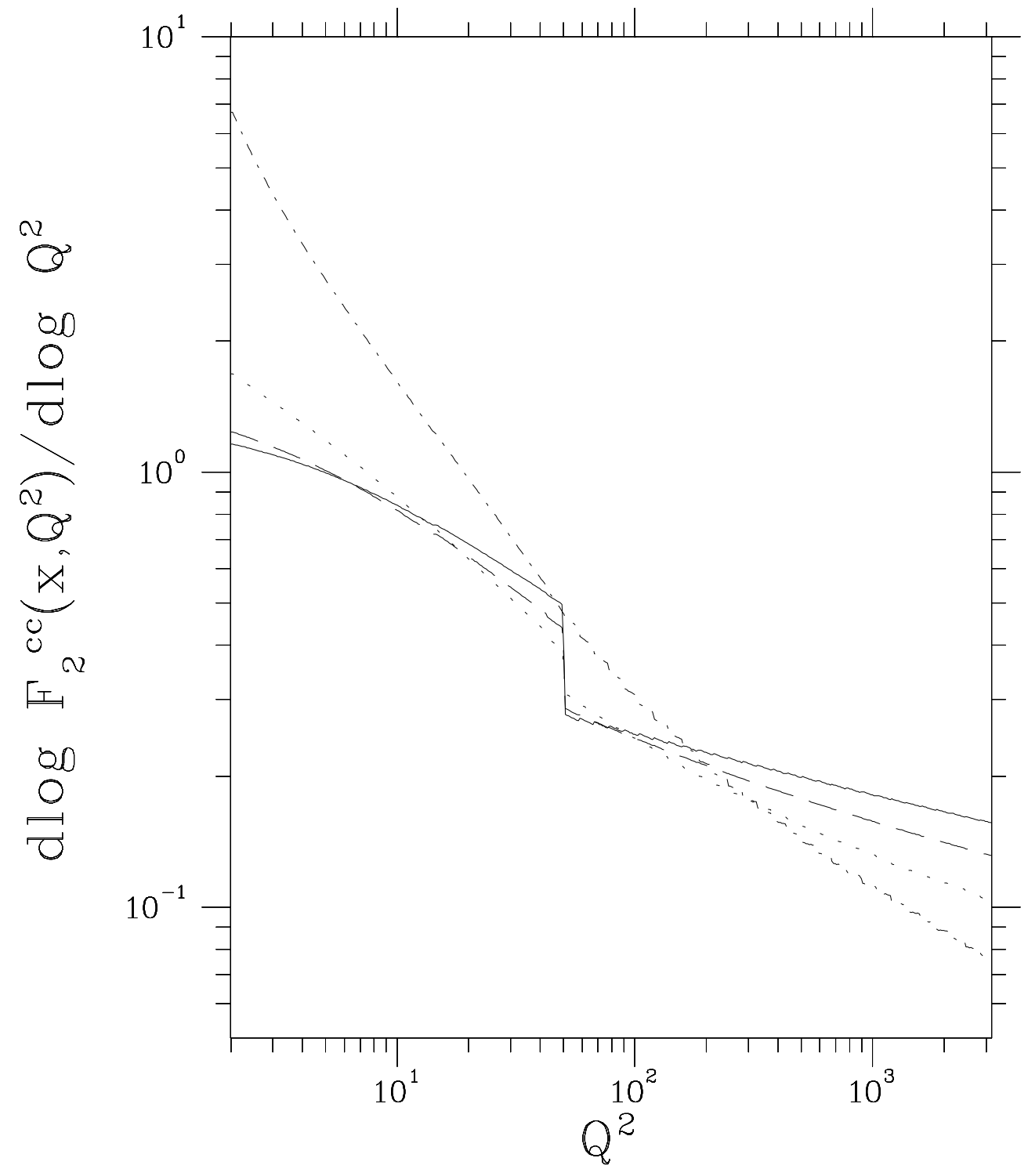

Figure 3d 


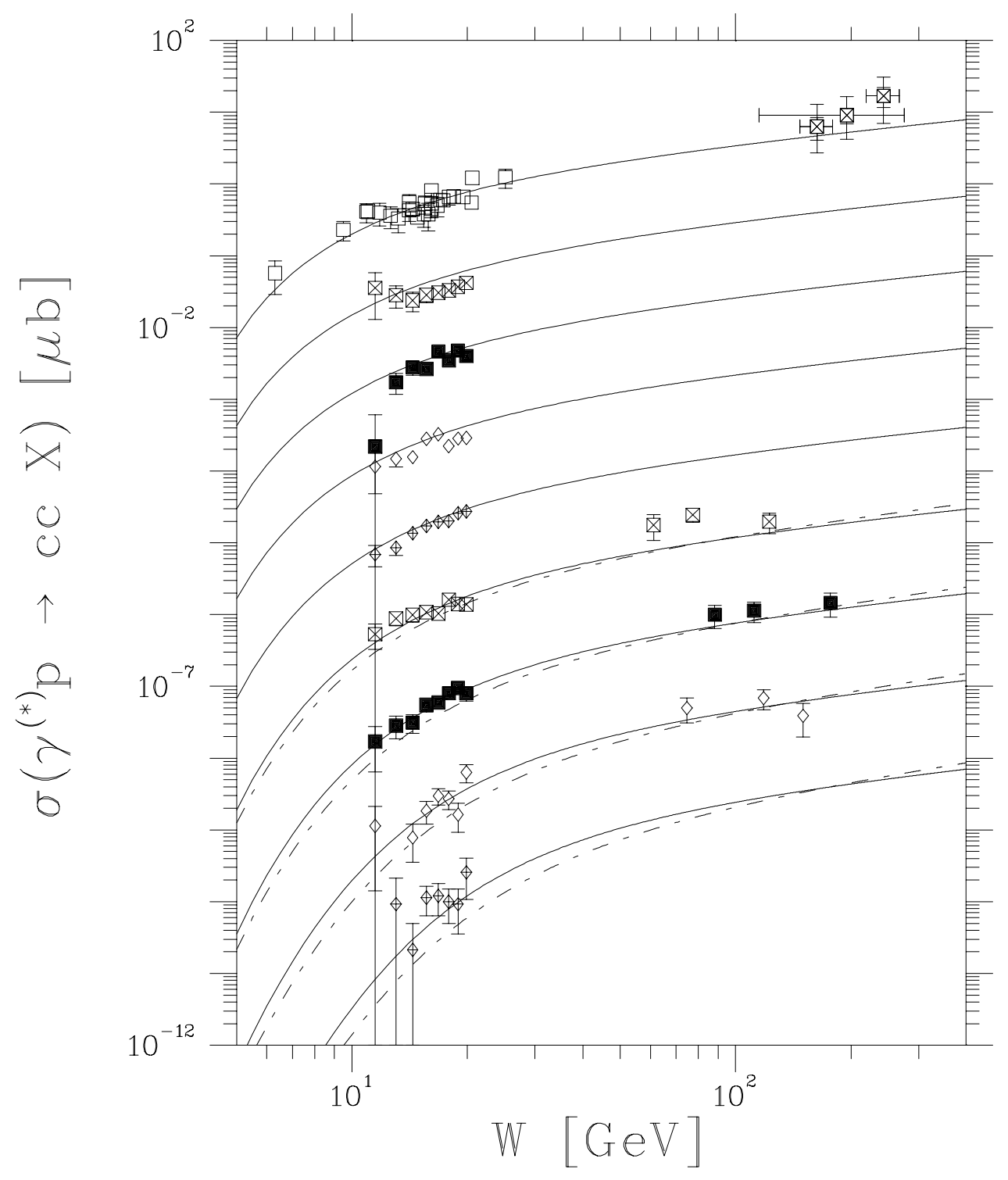

Figure 4 


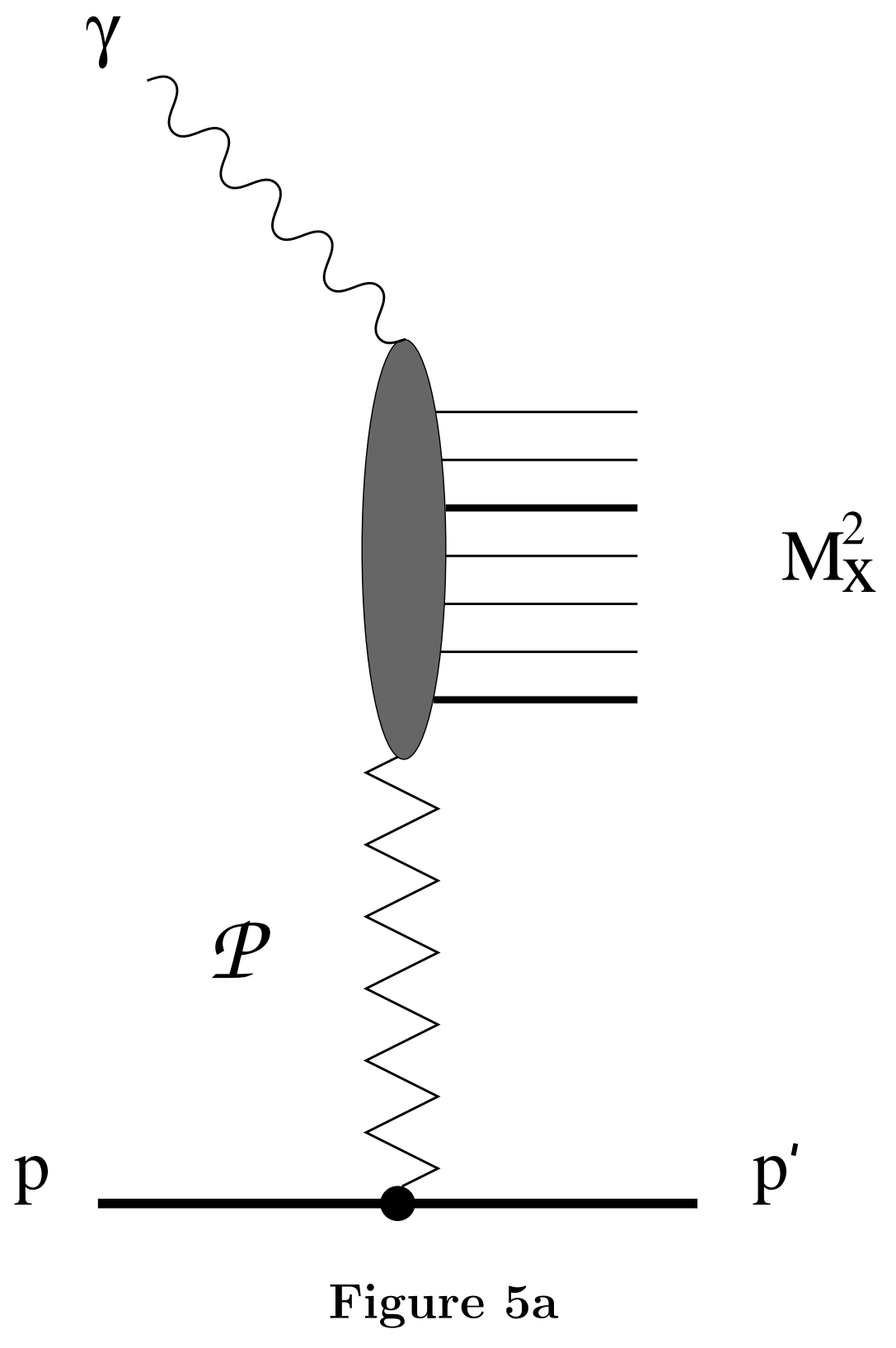




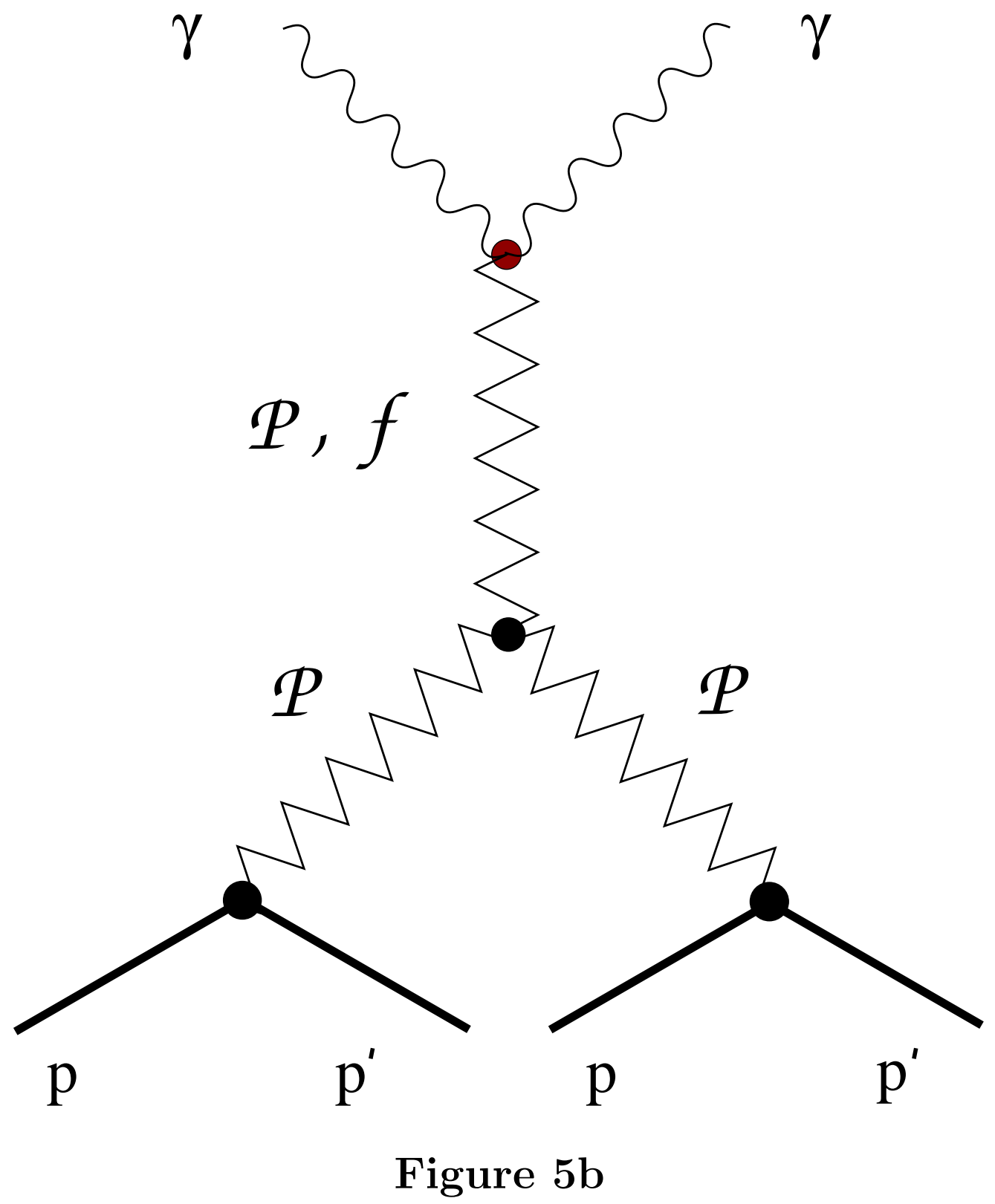




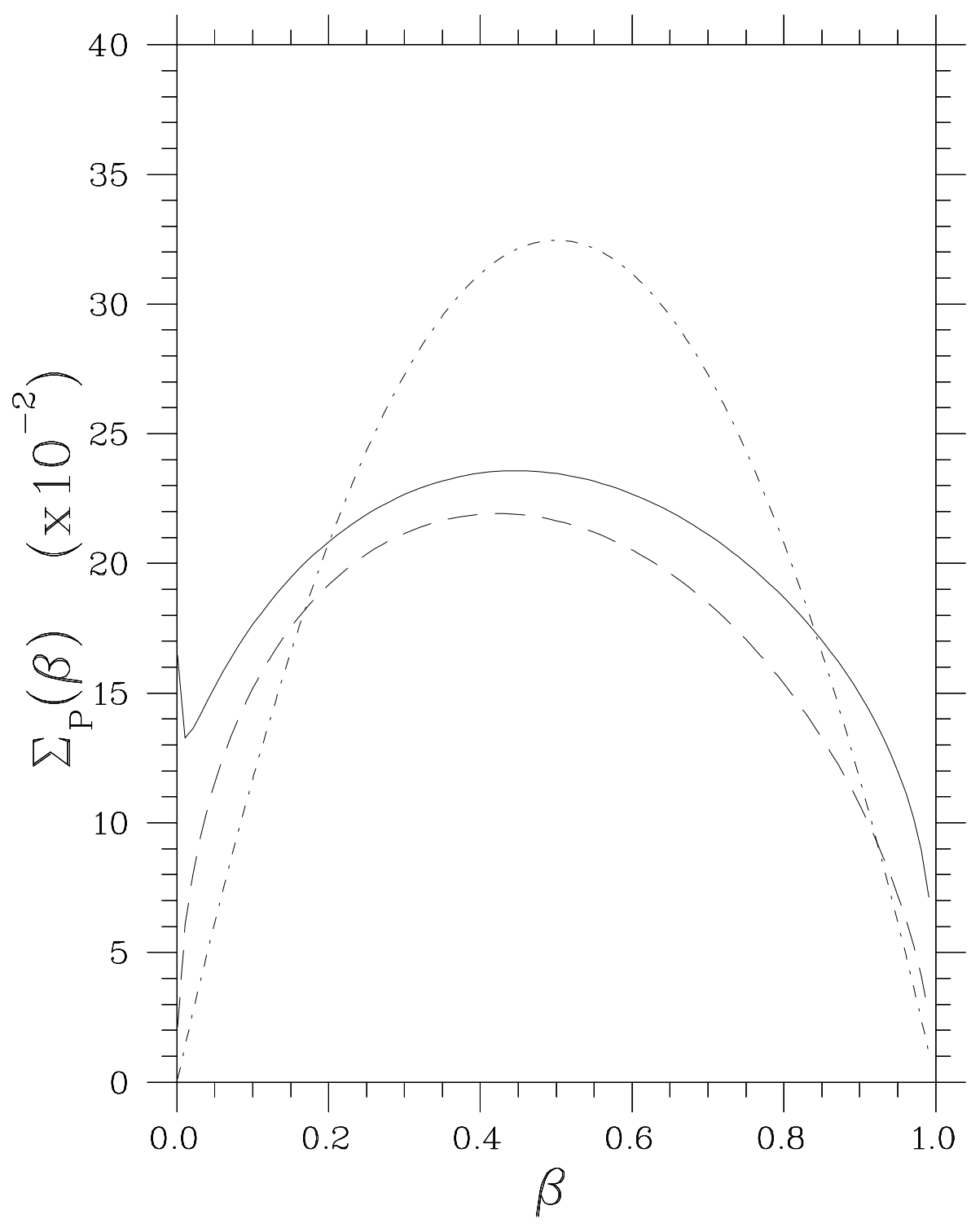

Figure 6a 


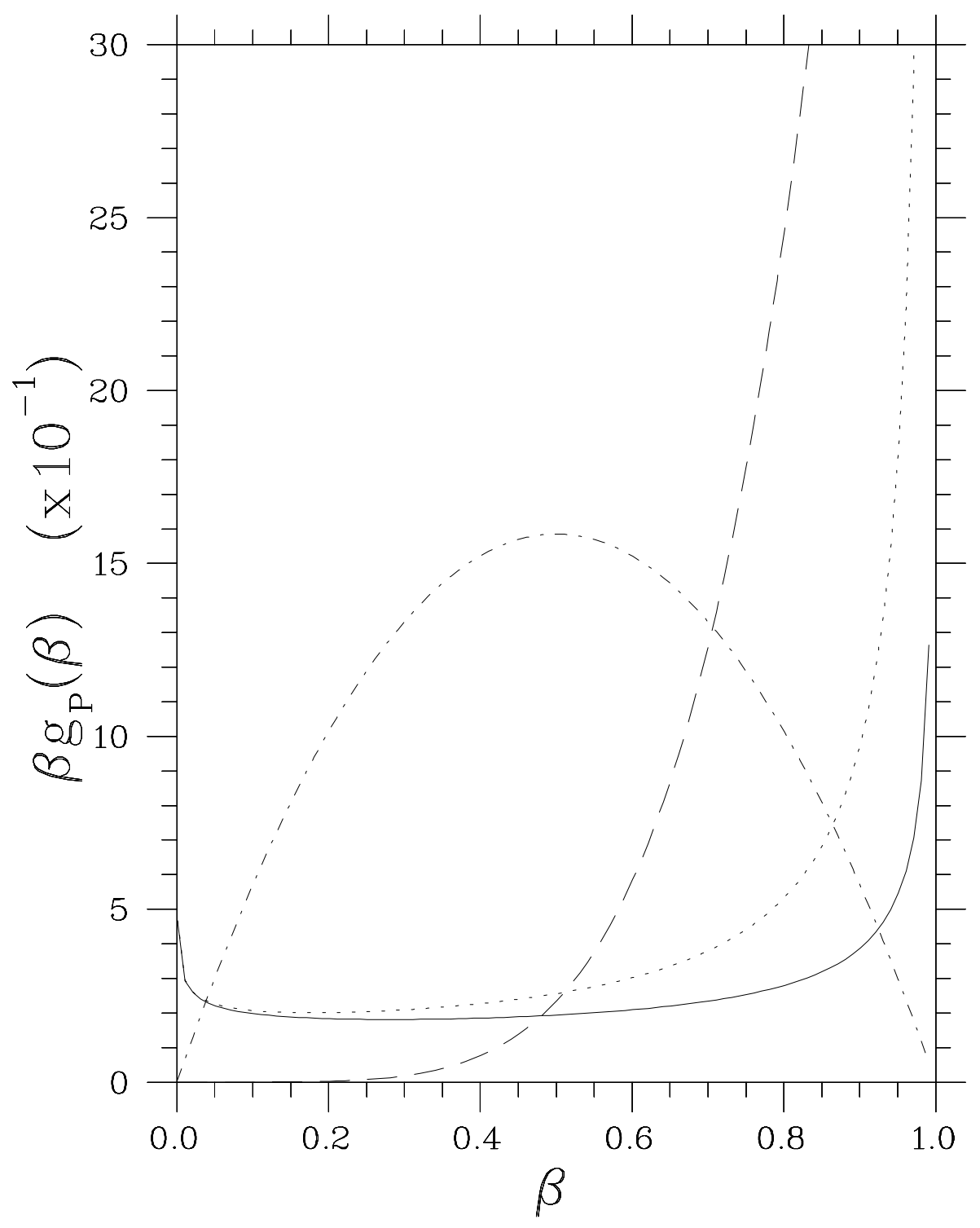

Figure $6 \mathrm{~b}$ 


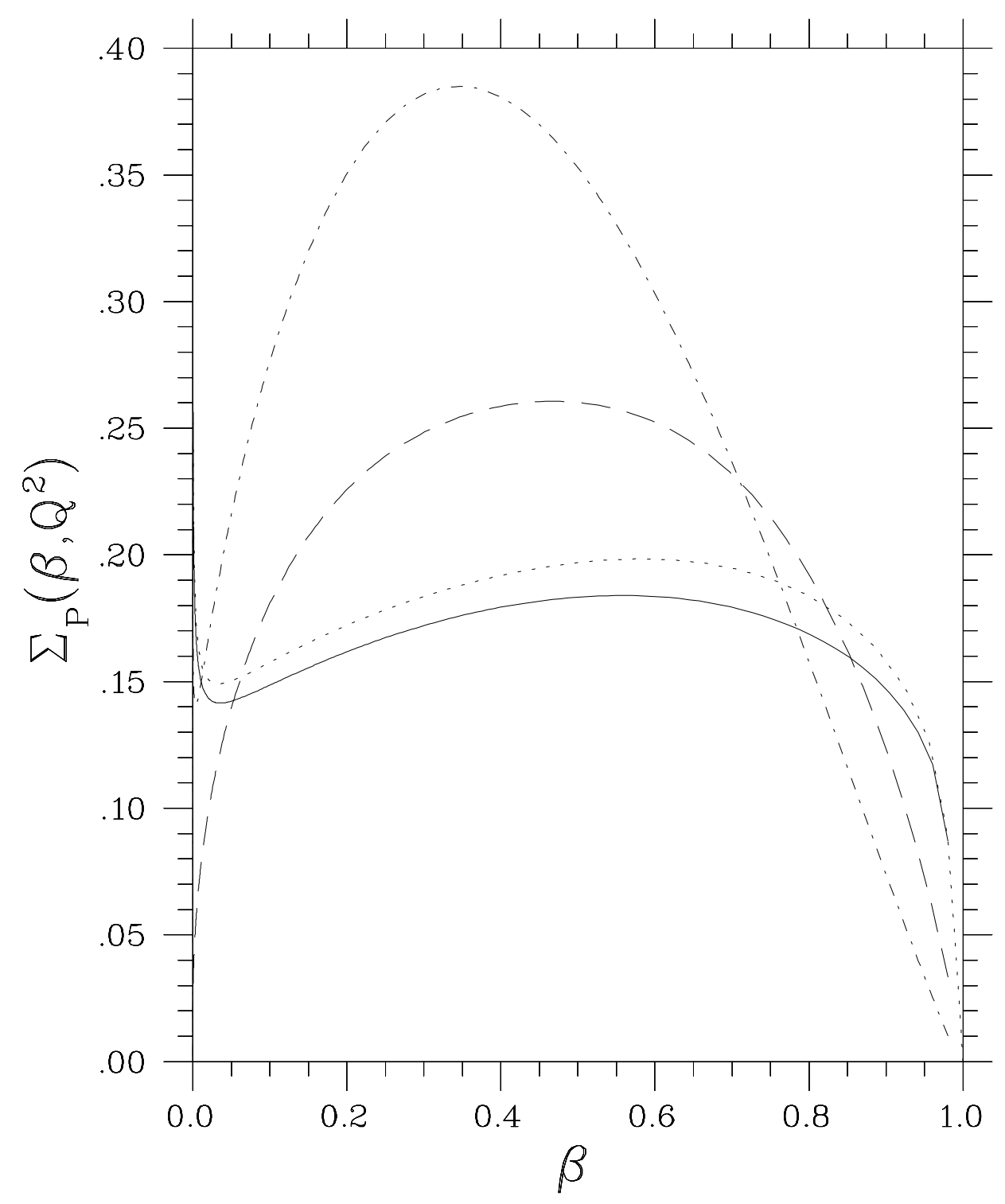

Figure 6c 


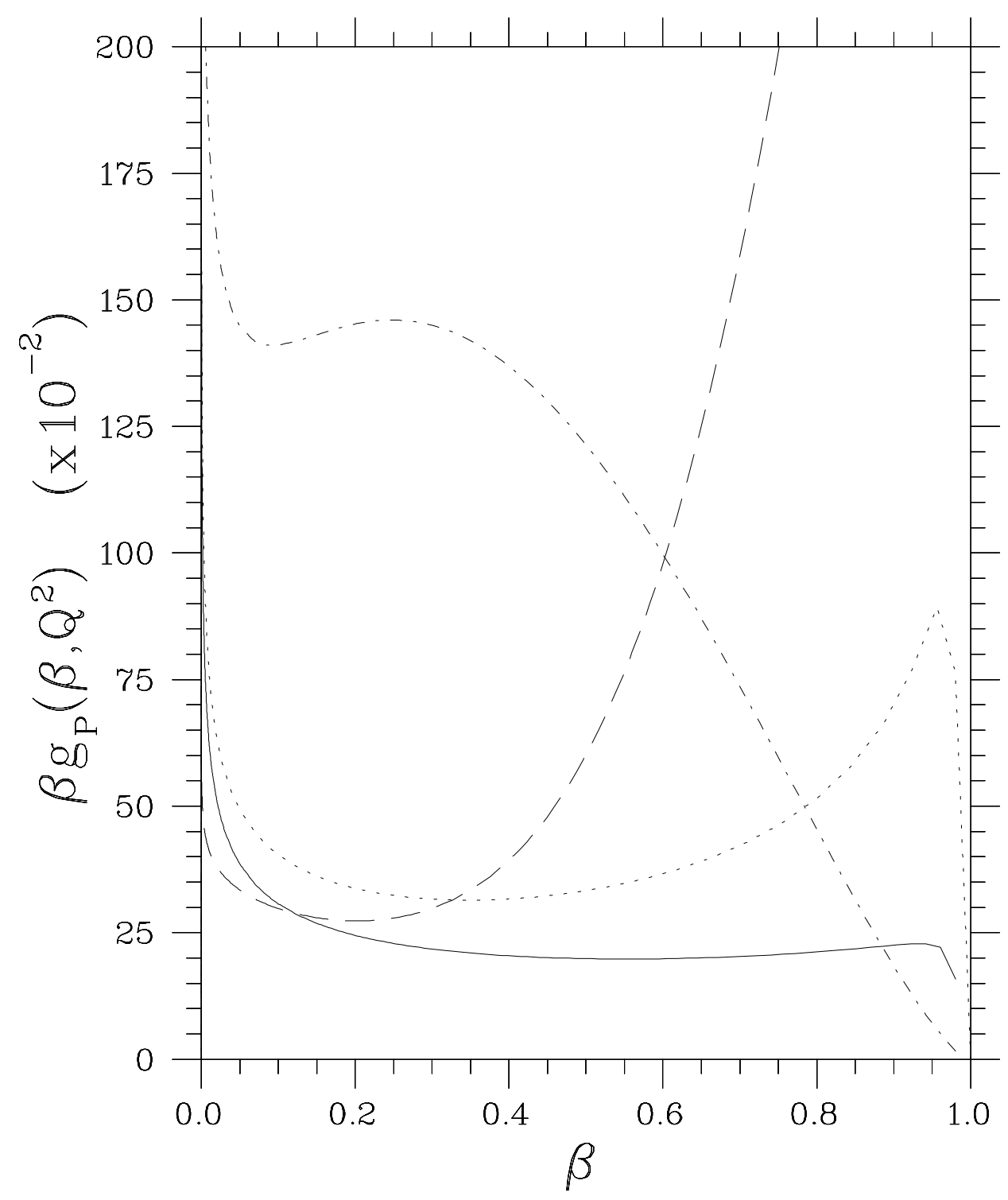

Figure 6d 


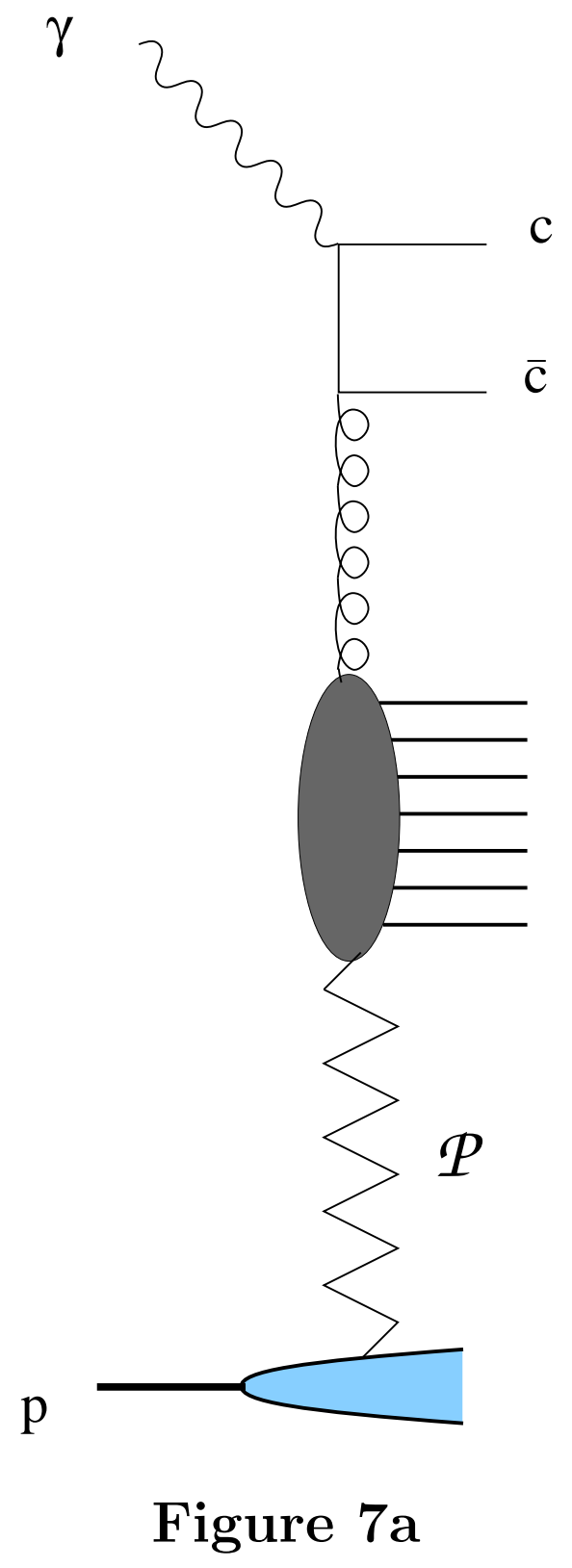




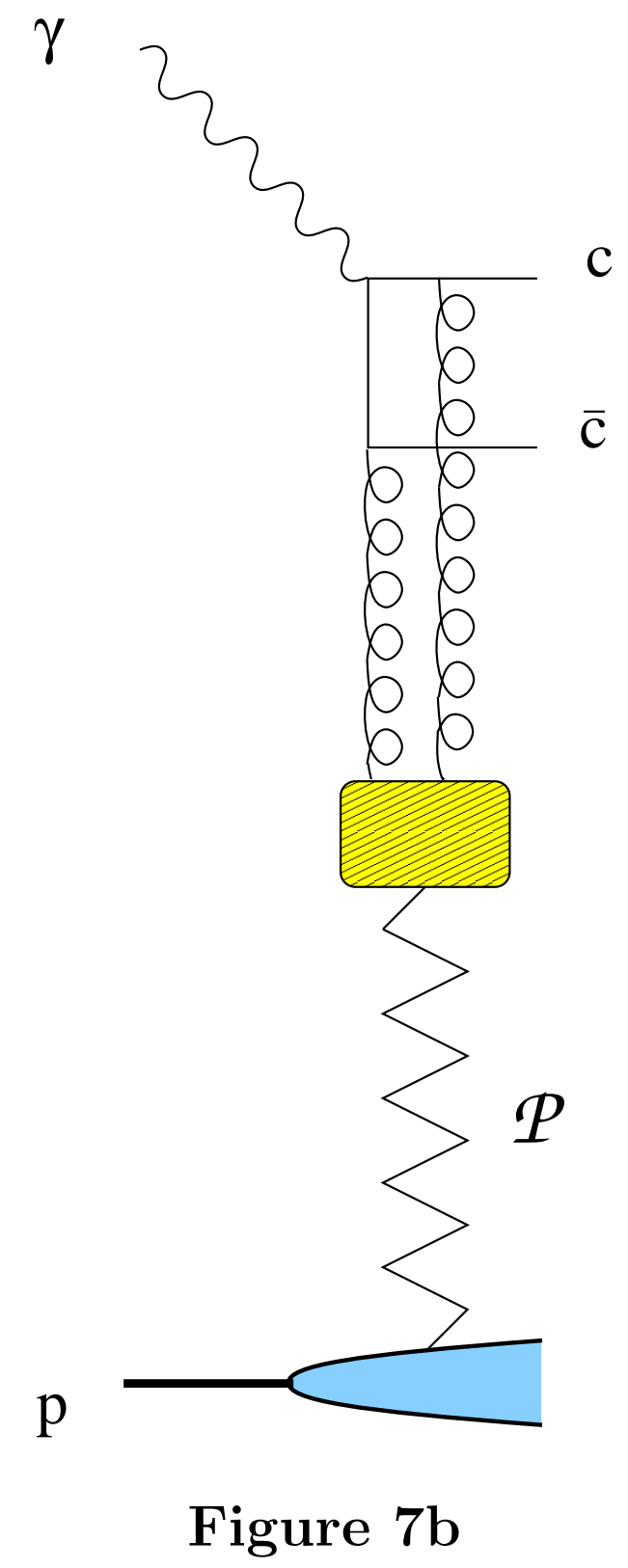




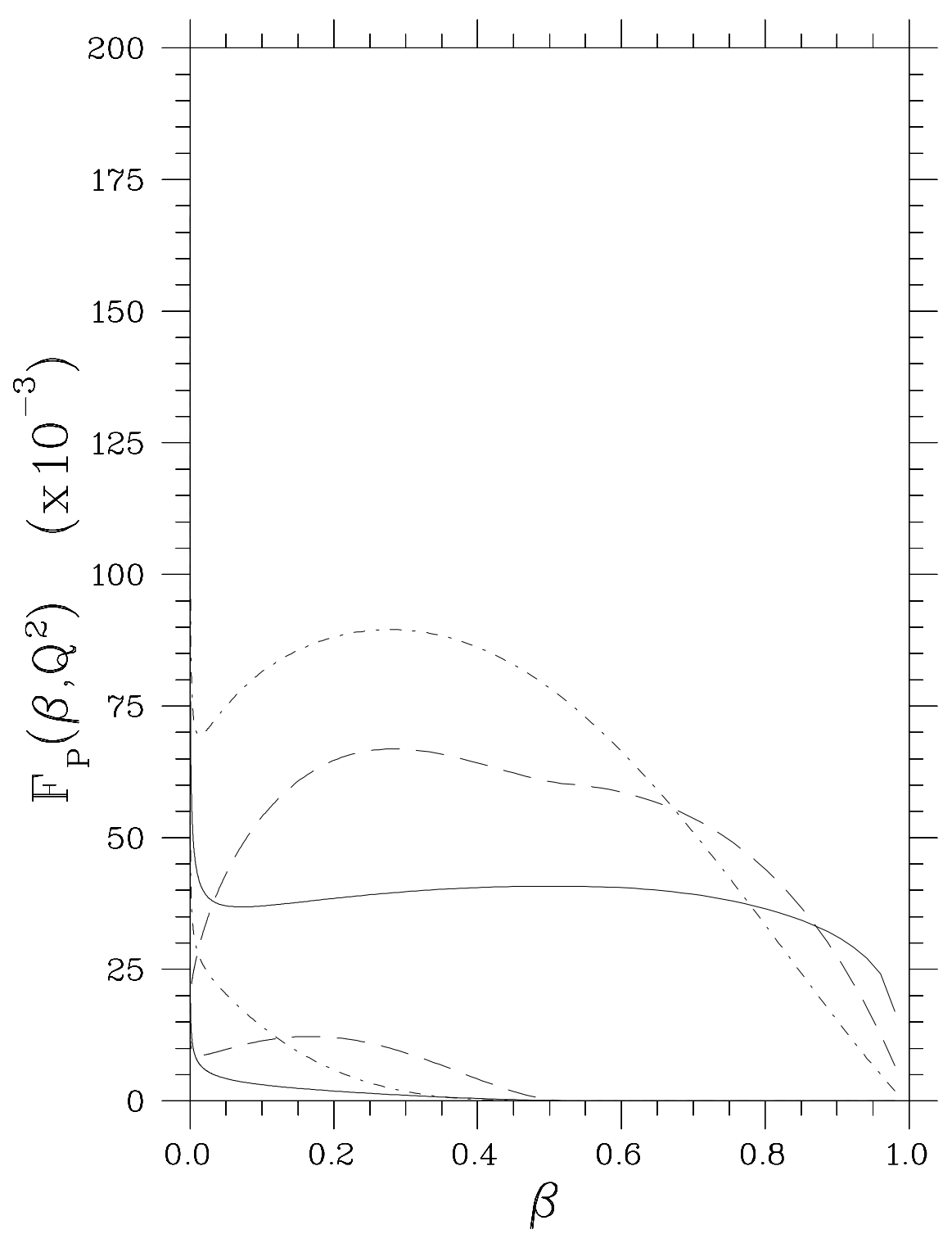

Figure 8a 


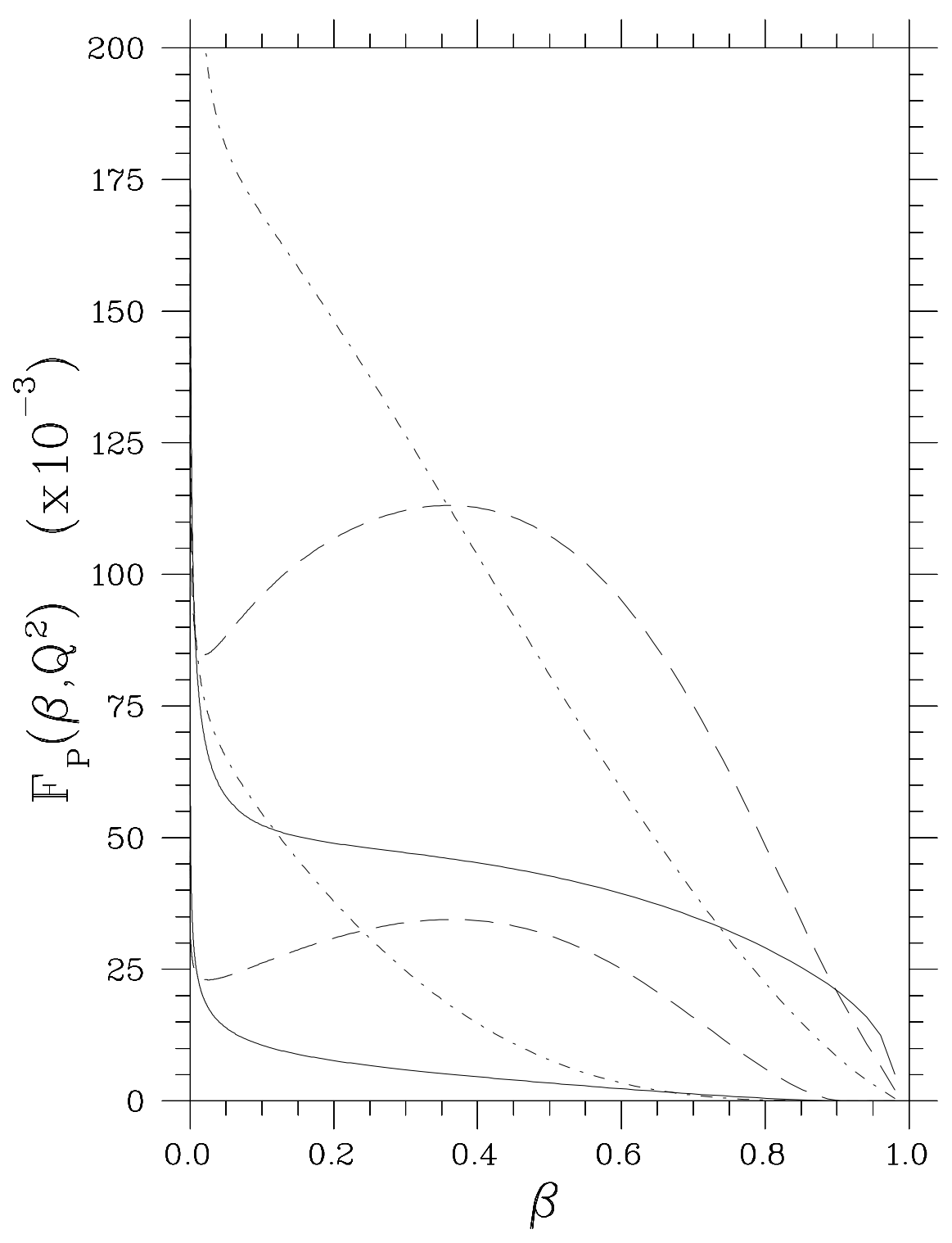

Figure 8b 


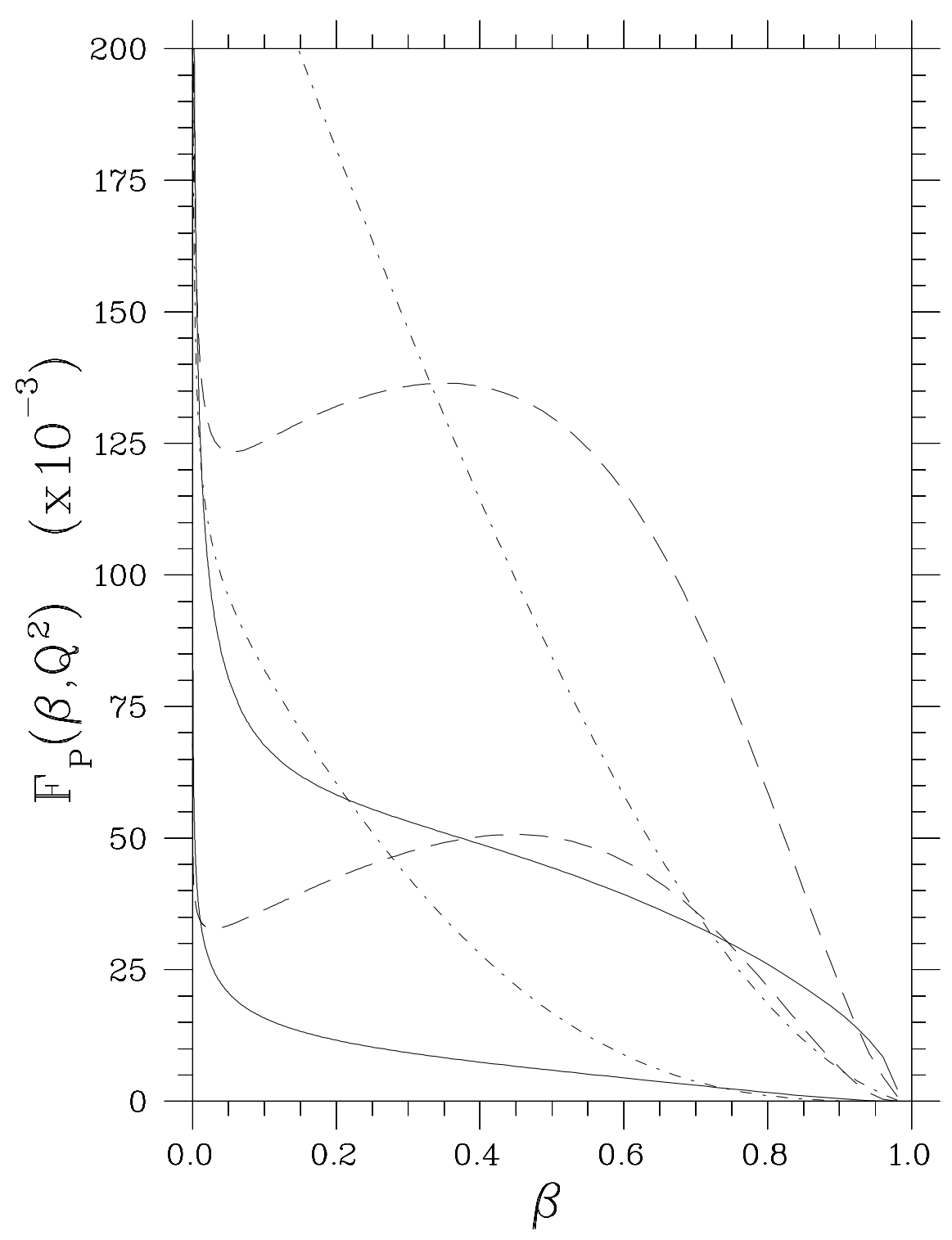

Figure 8c 


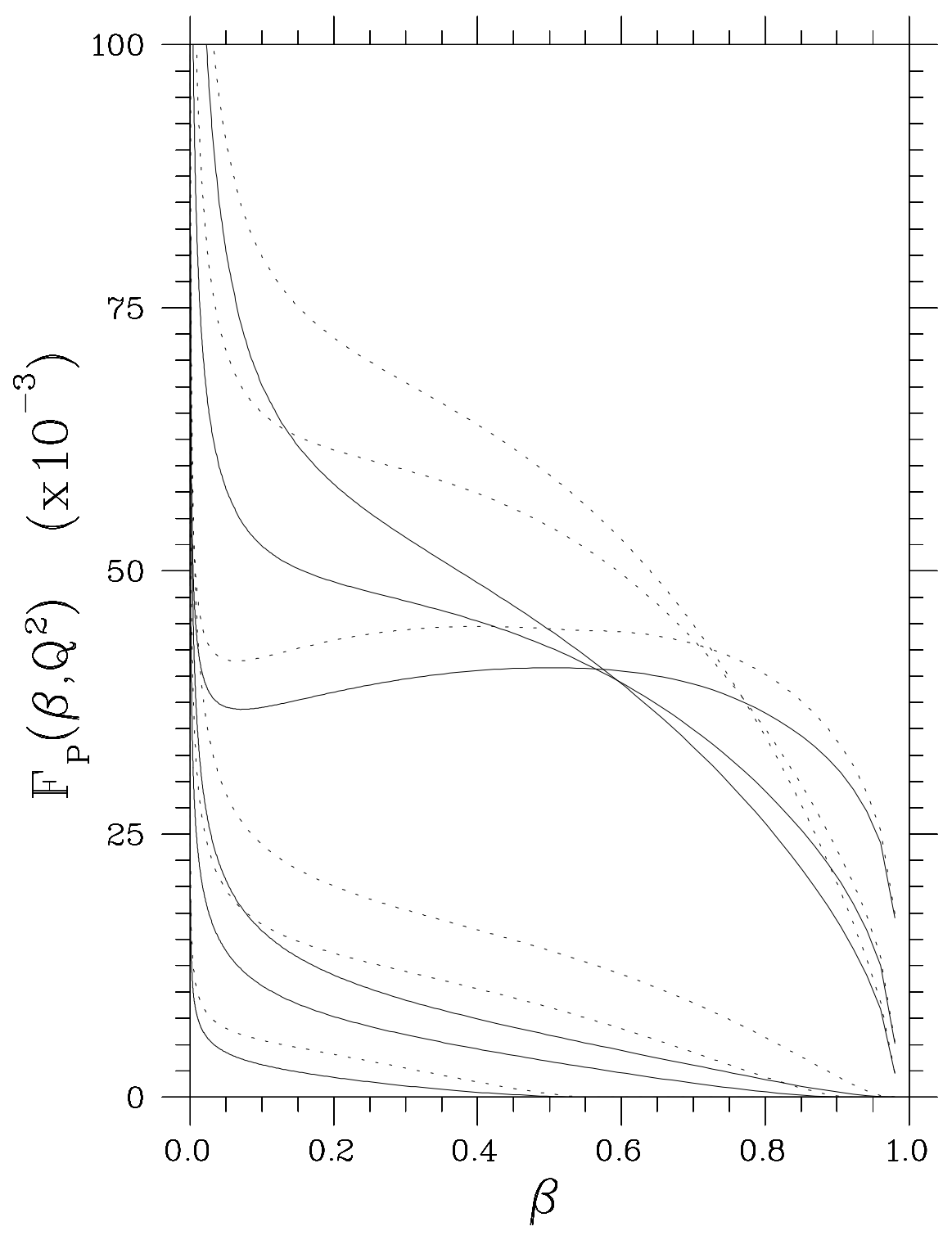

Figure 9 


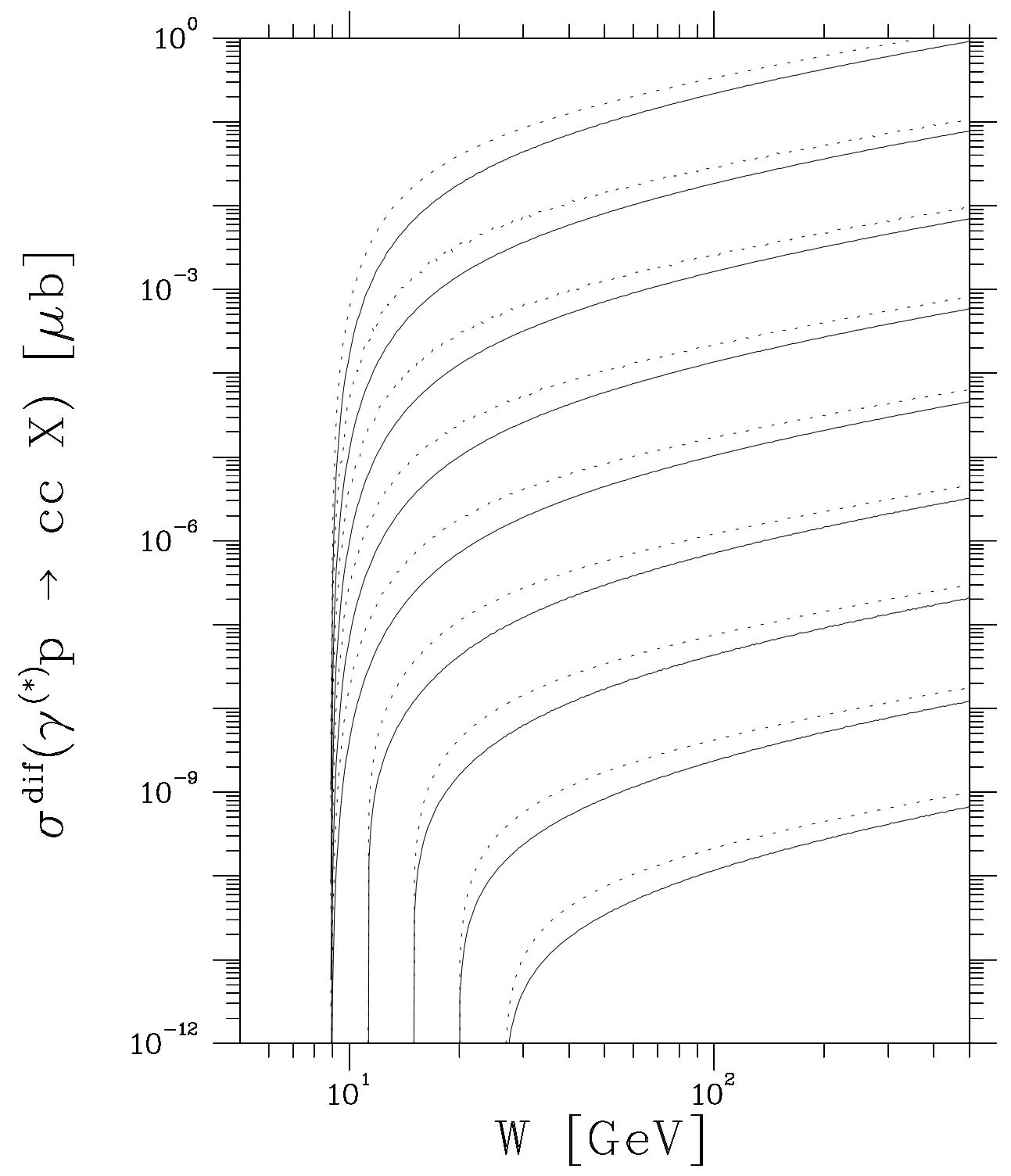

Figure 10 


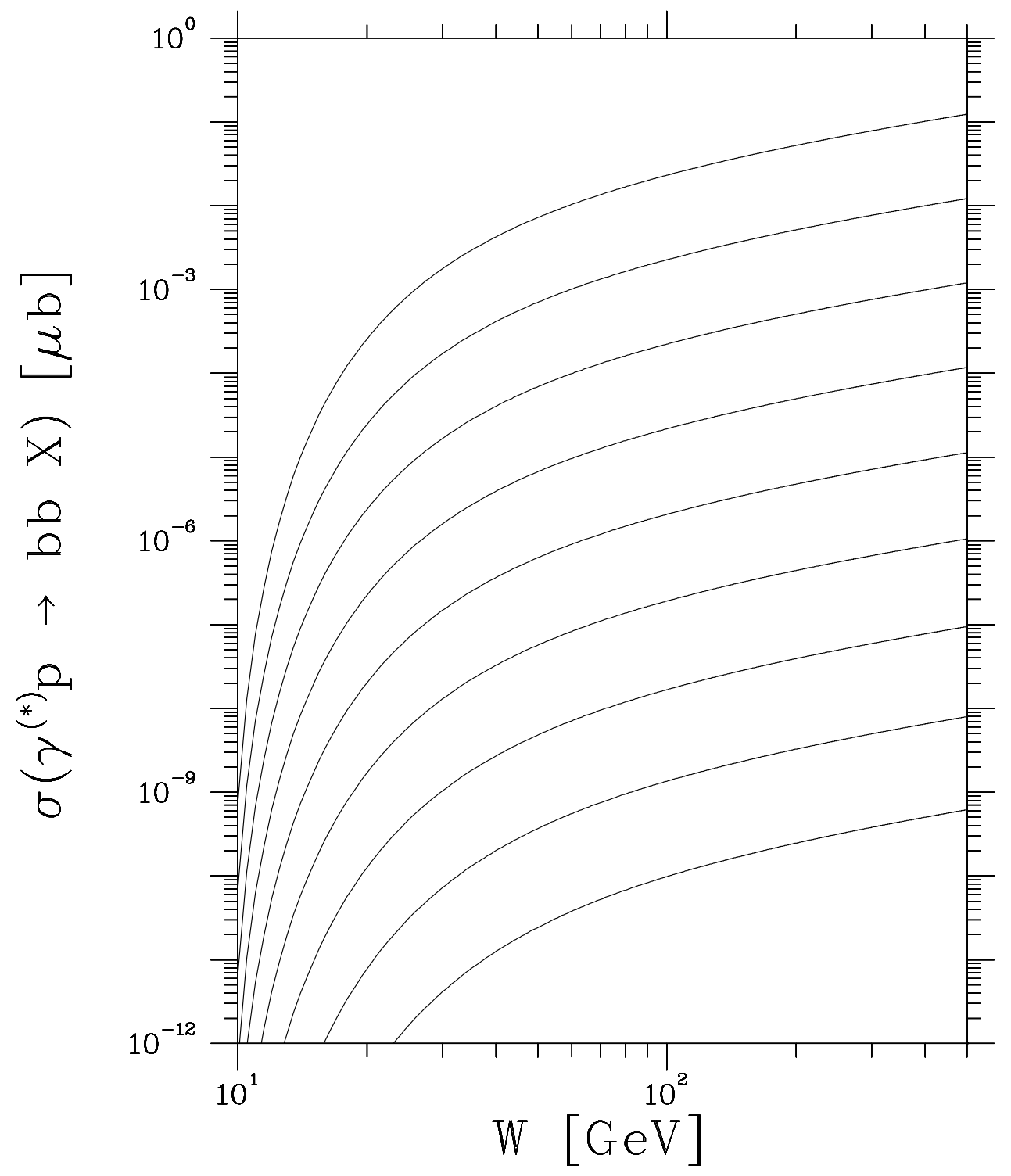

Figure 11 


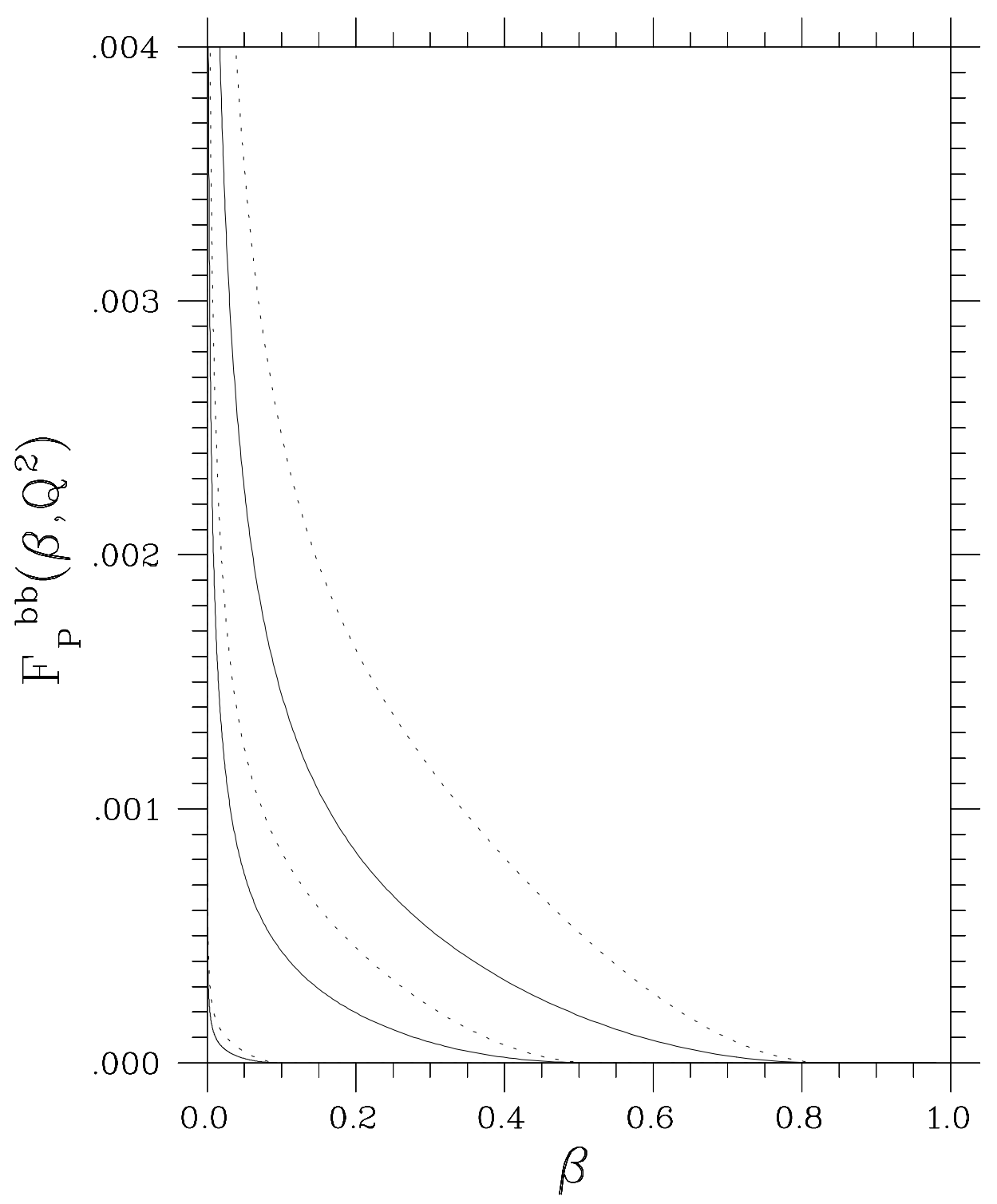

Figure 12 


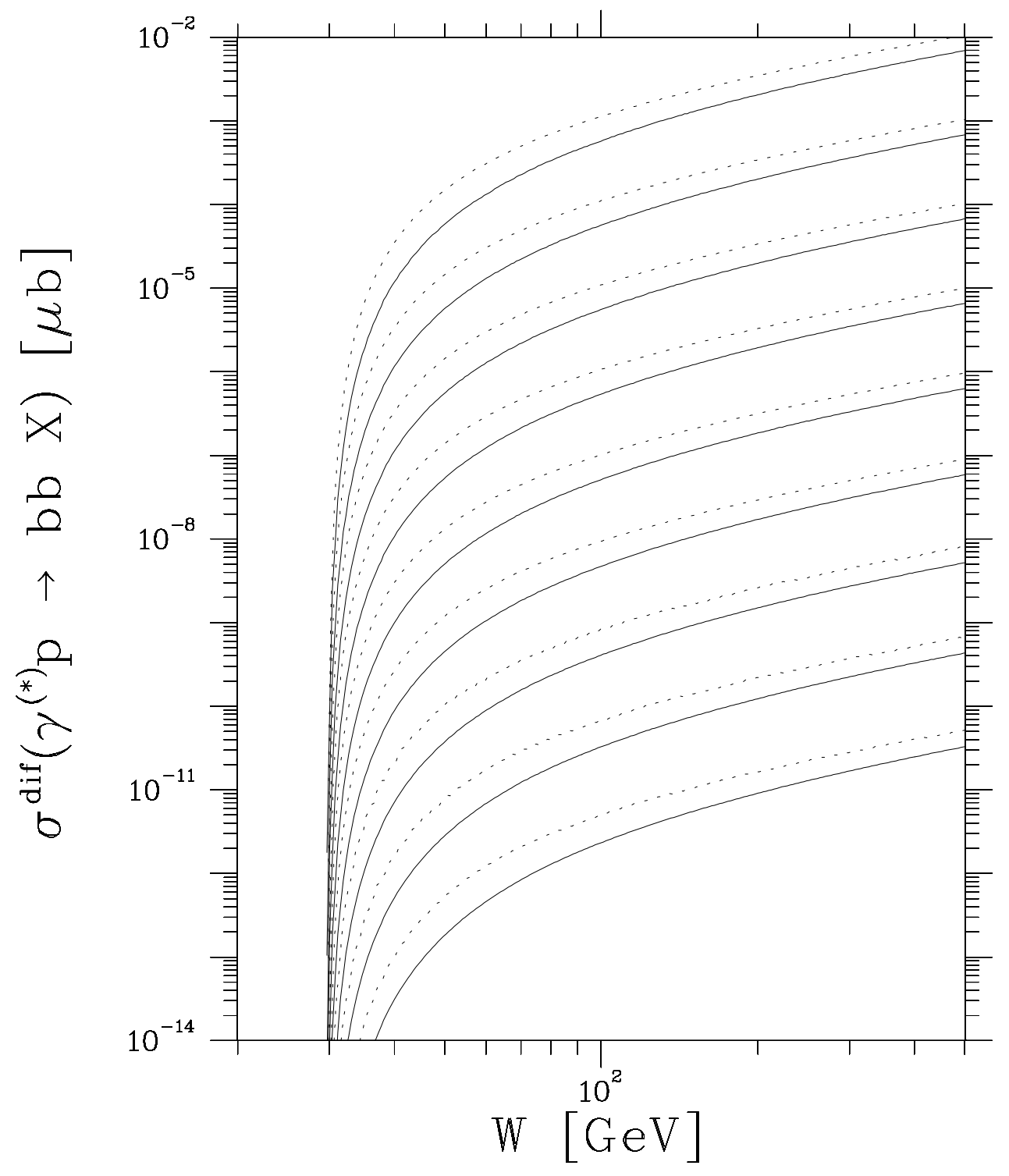

Figure 13 